\title{
ApoE4-carrying Human Astrocytes Oversupply Cholesterol into Neurons and Promote A $\beta$ Generation
}

\section{Se-In Lee}

DGIST: Daegu Gyeongbuk Institute of Science and Technology

\section{Woojin Jeong}

DGIST: Daegu Gyeongbuk Institute of Science and Technology

\section{Sukhee Cho}

DGIST: Daegu Gyeongbuk Institute of Science and Technology

\section{Hyein Lee}

DGIST: Daegu Gyeongbuk Institute of Science and Technology

\section{Yonghee Jang}

DGIST: Daegu Gyeongbuk Institute of Science and Technology

\section{Yuan-Ta Lin}

MIT: Massachusetts Institute of Technology

Li-Huei Tsai

MIT: Massachusetts Institute of Technology

Jinsoo Seo ( $\nabla$ jsseo@dgist.ac.kr)

Daegu Gyeongbuk Institute of Science and Technology https://orcid.org/0000-0001-6432-6964

\section{Research Article}

Keywords: Alzheimer's disease, human induced pluripotent stem cells, apolipoprotein E, cholesterol, lipid rafts

Posted Date: December 31st, 2020

DOI: https://doi.org/10.21203/rs.3.rs-122857/v2

License: (c) (1) This work is licensed under a Creative Commons Attribution 4.0 International License. Read Full License 


\section{ApoE4-carrying human astrocytes oversupply cholesterol into neurons and promote}

\section{A $\beta$ generation}

Se-In Lee ${ }^{1,4}$, Woojin Jeong ${ }^{1,4}$, Sukhee Cho ${ }^{1}$, Hyein Lee ${ }^{1}$, Yonghee Jang ${ }^{1}$, Yuan-Ta Lin²,3, Li-Huei Tsai ${ }^{2,3}$ and Jinsoo Seo ${ }^{1, *}$

${ }^{1}$ Department of Brain and Cognitive Sciences, Daegu Gyeongbuk Institute for Science and Technology, Daegu, 42988, South Korea.

2 Picower Institute for Learning and Memory, ${ }^{3}$ Department of Brain and Cognitive Sciences, Massachusetts Institute of Technology, Cambridge, MA 02139, USA.

${ }^{4}$ These authors contributed equally to this work

*Correspondence: jsseo@dgist.ac.kr

\section{SUMMARY}

The onset of Alzheimer's disease (AD) typically occurs later in life. Importantly, however, recent genetic analysis of patients and unaffected individuals revealed multiple genetic variants associated with late-onset $\mathrm{AD}$. One of the strongest genetic risk factors for $\mathrm{AD}$ is $\varepsilon 4$ allele of $A P O E$ encoding apolipoprotein (ApoE), which is predominantly expressed in glial cells. One of the overarching questions is whether and how this astrocyte-enriched risk factor initiates $\mathrm{AD}$-associated pathology in neurons such as $\mathrm{A} \beta$ accumulation and neurodegeneration. Here, we use human induced pluripotent stem cells (hiPSCs) from healthy individuals and isogenic cells in which the ApoE $\varepsilon 3$ allele was replaced with an $\varepsilon 4$ allele to generate human neurons and astrocytes. We then investigate the effect of 
astrocytic ApoE4 on the neuronal $A \beta$ production. We find that secretory factors in conditioned media from hiPSC-derived astrocytes carrying APOE4 significantly increased the levels of APP and A $\beta$ secretion in hiPSC-derived neurons. Increasing cholesterol levels in culture media mimicked the effects of ApoE4 ACM by inducing the formation of lipid rafts that potentially provide a physical platform for APP localization on the membrane. We further found that reducing cholesterol levels in ApoE4 ACM with $\mathrm{M} \beta C D$ abolished its effects on neuronal lipid raft expansion and $A \beta$ generation. Our study suggests that ApoE4 astrocytes contribute to amyloidosis by the expansion of lipid rafts and facilitate neuronal $\mathrm{A} \beta$ production through oversupply of cholesterol.

\section{KEYWORDS}

Alzheimer's disease, human induced pluripotent stem cells, apolipoprotein E, cholesterol, lipid rafts

\section{INTRODUCTION}

Alzheimer's disease (AD) is a progressive neurodegenerative brain disorder that accounts for the majority of cases of dementia ("2020 Alzheimer's disease facts and figures.," 2020). One of the major hallmarks of $\mathrm{AD}$ is the accumulation of amyloid- $\beta(\mathrm{A} \beta)$ in the brain (O'Brien and Wong, 2011; De Strooper and Karran, 2016). A $\beta$ is a fragment peptide derived from amyloid precursor protein (APP), which is highly expressed in neurons and is known to be important for neuronal development and function (O'Brien and Wong, 2011). $A \beta$ levels in the brain are tightly regulated by the balance between its production and clearance (Canter et al., 2016; De Strooper and Karran, 2016), and although A $\beta$ can be 
generated by non-neuronal cells, it is thought to be primarily produced by neurons under typical physiological conditions (Bu, 2009; O'Brien and Wong, 2011). To maintain a homeostatic environment, generated $A \beta$ is taken up by glial cells, such as astrocytes and microglia, to be degraded (Canter et al., 2016; Bu, 2009).

Genome-wide association studies have identified novel genetic risk factors associated with $\mathrm{AD}$, even in late-onset $\mathrm{AD}(\mathrm{LOAD})$ cases. In contrast with genetic variants in familial cases, many LOAD-associated variants are located in genes known to be enriched in glial cells (Canter et al., 2016; Liu et al., 2017). The precise AD-related phenotypes induced by these variants, and the mechanisms by which they arise, remain to be elucidated. Moreover, the reason as to why the timing of disease onset by these genetic factors is later than that induced by familial mutations located in APP, PSEN1, or PSEN2 remains uncertain. APOE4 is one of the strongest genetic risk factors for LOAD (Kunkle et al., 2019). ApoE is an apolipoprotein encoded by the $A P O E$ gene on chromosome 19, and is well known for its function in lipid transport by formation of lipoprotein complexes. In the central nervous system, ApoE is produced primarily by astrocytes, and its expression is upregulated in microglia under neurodegenerative conditions (Krasemann et al., 2017; Liu et al., 2017). There are three genotypes for ApoE in humans, including ApoE2, ApoE3, and ApoE4. Each genotype produces proteins that are considered to have structural differences according to amino acid sequences at 112 and/or 158 (ApoE2 - Cys112, Cys158; ApoE3 Cys112, Arg158; ApoE4 - Arg112, Arg158) (Liu et al., 2013). Although the difference in their sequence appears subtle, the translated proteins result in a significant difference in the risk for $\mathrm{AD}$. While the $\varepsilon 2$ allele is known to be protective, bearing $\varepsilon 4$ increases the risk of AD (Corder et al., 1993; Liu et al., 2013). 
A previous study using human induced pluripotent stem cells (iPSCs) from ApoE4 carriers suggested that ApoE4 contributes to amyloidosis by increasing $\mathrm{A} \beta$ secretion in ApoE4 neurons and decreasing $\mathrm{A} \beta$ clearance in ApoE4 astrocytes (Lin et al., 2018; Wang et al., 2018). However, the involvement and mechanisms by which ApoE4 astrocytes contribute to neuronal $\mathrm{A} \beta$ production remains to be determined. Here, using hiPSC-derived astrocytes and neurons carrying ApoE3 or ApoE4, we aimed to investigate whether ApoE4 astrocytes regulate neuronal $\mathrm{A} \beta$ production and if this regulation is mediated by abnormal cholesterol supply by ApoE4 astrocytes.

\section{RESULTS}

ApoE4 astrocyte-conditioned media increased APP expression and A $\beta_{42}$ secretion in hiPSC-derived neurons

To address the effect of ApoE4 astrocytes on neuronal APP expression and A $\beta$ generation, we utilized an iPSC line derived from healthy individuals carrying the ApoE3 allele and its isogenic line in which ApoE3 is converted to ApoE4 (Lin et al., 2018). Both ApoE3 and ApoE4 iPSC lines were differentiated in astrocytes or excitatory neurons as described previously (TCW et al., 2017; Zhang et al., 2013) with some modifications (Figure 1A), and immunostaining confirmed the identity of these cells (Supplementary Figure 1). In order to investigate whether secretory factors from ApoE4 astrocytes could affect neuronal A $\beta$ production, we generated healthy hiPSC-derived neurons carrying ApoE3 and cultured them in conditioned media from other healthy iPSC-derived astrocytes (ApoE3/E4 heterozygote) for five weeks. The media were then replaced with either ApoE3 or ApoE4 astrocyte conditioned media (ACM) and cultured for four days (Figure 1B). We found that 
ApoE4 ACM positively regulated the expression of APP in hiPSC-derived neurons carrying ApoE3 (Figure 1C, D). Immunoblotting from neuronal lysates revealed that APP levels were significantly increased by ApoE4 ACM (Figure 1E). We further measured the secreted levels of $A \beta_{40}$ and $A \beta_{42}$ and found that $A \beta_{42}$ secretion was significantly increased by ApoE4 ACM. These data show that secretory factors from ApoE4 astrocytes positively regulate neuronal APP expression and $\mathrm{A} \beta$ secretion.

Cholesterol positively regulated the formation of lipid rafts and APP expression in hiPSC-derived neurons

Accumulation of intracellular cholesterol in hiPSC-derived ApoE4 astrocytes compared to isogenic ApoE3 astrocytes has been recently reported (Lin et al., 2018; TCW et al., 2019), and Lin et al. further reported increased cholesterol secretion from ApoE4 astrocytes. Astrocytes supply cholesterol to neurons to support synapse formation and regulate membrane fluidity (Liu et al., 2013; Vance, 2012). Moreover, cholesterol, along with ganglioside and triglyceride, is a critical component of membrane lipid rafts, which provide a suitable platform for various membrane-bound proteins, including glutamate receptors. APP and its processing secretases, $\beta$ - and $\gamma$-secretase, are also known to be located in lipid rafts, while $\alpha$-secretase is mainly expressed in non-lipid rafts (Cheng et al., 2007; Raffai and Weisgraber, 2003).

Previous studies have shown that increasing cholesterol in the membrane induced the formation of lipid rafts and increased A $\beta$ production (Cossec et al., 2010; Marquer et al., 2014). Therefore, we hypothesized that increased levels of cholesterol in ApoE4 ACM could be a key factor in upregulating APP and its processing by facilitating the formation 
of lipid rafts. First, the conditions were optimized to regulate environmental cholesterol levels by treating neurons with cholesterol or methyl $\beta$-cyclodextrin $(\mathrm{M} \beta \mathrm{CD}$; to deplete cholesterol) in a rat primary neuron culture system (Supplementary Figure 2A). We found that treatment with $20 \mu \mathrm{M}$ of cholesterol for four days was sufficient to increase neuronal cholesterol, as visualized by filipin III staining, which is naturally fluorescent upon cholesterol binding (Supplementary Figure 2B, C). We then measured the levels of lipid rafts by staining for cholera toxin B (CTX-B), a well-known lipid raft marker, and found a significant increase in lipid rafts following cholesterol treatment (Supplementary Figure 2D-E). APP expression was also increased in cholesterol-treated rat primary neurons (Supplementary Figure 2F). There was no reduction in both neuronal cholesterol and lipid rafts following $\mathrm{M} \beta \mathrm{CD}$ treatment in rat primary neurons (Supplementary Figure 2B-E), which could be due to the homeostatic mechanism of neurons to compensate for the loss of intracellular cholesterol. To address whether cholesterol treatment is sufficient to mimic the effect of ApoE4 ACM on neuronal APP expression and A $\beta$ secretion, as shown in Figure 1C-F, we treated hiPSC-derived neurons with cholesterol or M $\beta C D$ (Figure 2A). The upregulation of cholesterol in neurons by exogenous cholesterol treatment was confirmed by filipin III staining (Figure 2B, C). Consistent with the observation in rat primary neurons, $\mathrm{M} \beta \mathrm{CD}$ treatment did not alter the levels of cholesterol in hiPSC-derived neurons (Figure 2B, C). We then measured levels of lipid rafts in neurons and found that cholesterol treatment increased the area of CTX-B signals without affecting intensity, suggesting the expansion of lipid rafts (Figure 2D, E). We also measured APP levels in neurons and found significant upregulation of APP expression by cholesterol treatment, due to increased area of APP signals rather than intensity (Figure 2F), which is consistent 
with rat primary neurons treated with cholesterol (Supplementary Figure 2F). We further found that the co-localization of APP and CTX-B was significantly increased by cholesterol treatment in both hiPSC-derived neurons and rat primary neurons (Figure 2G, Supplementary Figure 2G). To determine whether APP upregulation is caused simply by the expansion of lipid rafts or if more APP is recruited to the given area of lipid rafts, we measured the intensity of APP in the CTX-B/APP co-localized area. The data showed that there was no alteration in APP intensity in these regions (Figure 2G, Supplementary Figure $2 \mathrm{G})$, suggesting that increased APP expression by extracellular cholesterol supply is mainly due to the increased area of lipid rafts.

\section{Cholesterol in ApoE4 ACM increased the formation of lipid rafts in hiPSC-derived}

\section{neurons}

To investigate whether the effects of ApoE4 ACM on neuronal cholesterol levels and lipid raft formation were due to cholesterol oversupply, we measured cholesterol levels in ApoE3 and ApoE4 ACM. Consistent with a previous report (Lin et al., 2018), we found higher cholesterol levels in ApoE4 ACM than in ApoE3 ACM (Figure 3A). We then measured filipin III in hiPSC-derived neurons cultured with conditioned media from either ApoE3 or ApoE4 astrocytes as described in Figure 1B, and ApoE4 ACM-treated neurons displayed increased filipin III signals (Figure 3B, C). We also found that the area and total levels of CTX-B were significantly increased compared to those of ApoE3 ACM-treated neurons (Figure 3D-E). To determine whether the cholesterol in ApoE4 ACM is the major cause of the upregulation of lipid rafts and APP in neurons, we added M $\beta C D$ to ApoE4 $\mathrm{ACM}$ during neuronal culture (Figure $3 \mathrm{~F}$ ) and found that the upregulation of neuronal 
cholesterol by ApoE4 ACM was significantly attenuated by $\mathrm{M} \beta \mathrm{CD}$, potentially due to its scavenging effect toward exogenous cholesterol (Figure $3 \mathrm{G}, \mathrm{H}$ ). The addition of $\mathrm{M} \beta \mathrm{CD}$ to ApoE4 ACM abolished the ApoE4 ACM-induced increase in lipid raft expansion (Figure 3I, J).

Reducing cholesterol attenuated ApoE4 ACM-induced APP upregulation and AB 42 secretion in hiPSC-derived neurons

To determine whether cholesterol in ApoE4 ACM was the major cause for the upregulation of APP and its metabolism to produce A $\beta$ in hiPSC-derived neurons, we added $\mathrm{M} \beta \mathrm{CD}$ to ApoE4 ACM during neuronal culture (Figure 4A). As shown in Figure 1E, neurons cultured with ApoE4 ACM showed increased expression of APP compared to those cultured with ApoE3 ACM. However, in the presence of M $\beta C D$, ApoE4 ACM was not able to induce significant upregulation of APP. Increased co-localization of lipid rafts and APP by ApoE4 ACM was also abolished in neurons treated with M $\beta C D$ (Figure 4BE). Furthermore, $M \beta C D$ treatment inhibited the ApoE4 ACM-induced increase in $A \beta_{42}$ secretion in hiPSC-derived neurons (Figure 4F). Taken together, these data suggest that an excess supply of cholesterol is responsible for ApoE4 ACM-mediated neuronal A $\beta_{42}$ overproduction.

\section{DISCUSSION}

Abnormal neuronal cholesterol levels have been linked to AD-related pathology both in vitro and in vivo. For example, increased levels of cholesterol were observed in AD brain samples, and the severity of pathology was correlated with cholesterol levels (Cutler et al., 
2004; Lazar et al., 2013). Inhibition of cholesterol efflux by reducing expression of CYP46A1, a cholesterol 24S-hydroxylase, in a neuron-specific manner was shown to result in cognitive deficits and neuronal death in wild-type mice. The study further showed the recruitment of APP to lipid rafts and A $\beta$ upregulation prior to neuronal death in both wildtype and APP23 mice (Djelti et al., 2015). Here, we showed that ApoE4 bearing astrocytes increased cholesterol supply to neurons, leading to the upregulation of APP and A $\beta$ secretion in a paracrine manner. Application of cholesterol recapitulated the effects of ApoE4 ACM on neurons, and the effects of ApoE4 ACM were abolished by M $\beta C D$. These data revealed the major contribution of secretory cholesterol from ApoE4 astrocytes to lipid raft expansion and APP expression, which promotes $A \beta_{42}$ secretion in neurons.

A recent study showed that $\mathrm{CHO}$ cell lines expressing familial AD-associated PSEN1 $\Delta \mathrm{E} 9$ display increased levels of cholesterol, which leads to the enrichment of APP in lipid rafts (Cho et al., 2019). Similarly, a previous study suggested binding between cholesterol and $\beta$-secretase-derived APP C-terminal fragment ( $\beta$-CTF) (Beel et al., 2008). These data suggest the active role of cholesterol in recruiting APP to lipid rafts. Here, we found that cholesterol oversupply by ApoE4 astrocytes increases the levels of APP in lipid rafts. Although we did not find a difference in APP intensity (local clustering density) in lipid rafts by extracellular cholesterol supply (Figure 2G and Supplementary Figure 2G), neurons cultured in ApoE4 ACM displayed increased APP intensity in lipid rafts, which was abolished by $\mathrm{M} \beta \mathrm{CD}$ treatment (Figure 4D). As cholesterol was shown to increase the proximity between APP and $\beta$-secretase 1 (BACE1) in the membrane (Marquer et al., 2011), further studies are required to examine the expression pattern of $\beta$ - and $\gamma$-secretases on lipid rafts in neurons when they are cultured with ApoE3 or ApoE4 ACM. 
Cholesterol was also shown to increase A $\beta$ production through the facilitation of APP endocytosis to endosomes (Cossec et al., 2010). The low $\mathrm{pH}$ environment in endosomes increases BACE1 activity to facilitate APP cleavage and produce $\beta$-CTF. Increased size and/or number of early endosomes were found in the brains of AD patients as well as in multiple AD model systems. Moreover, cholesterol loading to the neuronal plasma membrane was shown to result in enlarged endosomes (Marquer et al., 2014). In the current study, for the first time, we revealed the impact of ApoE4 astrocytes on neuronal cholesterol and lipid rafts that affect $A \beta$ production. Further studies are required to determine whether astrocytic ApoE4 regulates APP processing only in lipid rafts or in endosomal compartments, that is whether cholesterol only affects membrane-originated $A \beta$ production and/or facilitates APP endocytosis and subsequent $A \beta$ generation in endosomes.

Lipid rafts provide a platform for various neuronal membrane proteins and are important for synaptic functions (Hering et al., 2003). Previously, cholesterol was shown to promote synapse maturation, whereas depletion of cholesterol significantly reduced lipid raft domain and synapses (Hering et al., 2003; Mauch et al., 2001). The positive correlation between neuronal activity and $A \beta$ production has been supported by multiple studies (Bero et al., 2011; Das et al., 2013), and the expansion of lipid rafts by ApoE4 ACM could also contribute to the upregulation of $A \beta$ by increasing neuronal activity.

In addition to $A \beta$ production, $A \beta$ clearance can be manipulated by the ApoE4 ACM. Cholesterol accumulation in cellular organelles such as lysosomes has been found to increase intracellular A $\beta$ levels (Yamazaki et al., 2001). Therefore, it would be interesting to determine whether increased cholesterol supply from ApoE4 astrocytes induces 
lysosomal cholesterol accumulation and impairs lysosomal activity and $\mathrm{A} \beta$ degradation in neurons.

ApoE4, the strongest genetic risk factor for LOAD, has recently been shown to have detrimental effects on astrocytes, including endocytic defects and impaired homeostatic functions (Fernandez et al., 2019; Lin et al., 2018; Narayan et al., 2020). It is not clear, however, whether altered astrocytic properties could affect neighboring neurons and induce AD-associated pathology. Here, we revealed that ApoE4 astrocytes could regulate neuronal APP metabolism to initiate amyloidosis through cholesterol oversupply (Supplementary Figure 3). This study provides new insight into the contribution of ApoE4 and astrocytes to amyloidosis in $\mathrm{AD}$, and the importance of regulating astrocytic $\mathrm{ApoE}$ isotypes.

\section{EXPERIMENTAL PROCEDURES}

\section{iPSCs culture}

The use of human iPSCs was approved by the Institutional Review Board (IRB) of Daegu Gyeongbuk Institute for Science and Technology (Permit Number: DGIST-190829-BR071-01). ApoE3 iPSC line is generated from the Coriell Institute's fibroblast line derived from healthy individuals (age 75, female; \#AG09173) by Dr. Yankner Laboratory at Harvard Medical School (Meyer et al., 2019). ApoE4 isogenic line is generated from this ApoE3 iPSC line as previously described (Lin et al., 2018). For ApoE3/E4 heterozygous astrocyte conditioned media (ACM), an iPSC line derived from a healthy individual (age 22, female; \#GM23720) was obtained from the Coriell Institute. iPSCs were maintained 
on matrigel (Corning 354277)-coated plate in mTeSR1 media (Stemcell) at $37^{\circ} \mathrm{C}$ with $5 \%$ $\mathrm{CO}_{2}$ conditioned incubator.

\section{Neural progenitor cells differentiation}

The iPSCs were cultured with mTeSR 1 media on the matrigel coated 6-well plate. When cell density was $100 \%$ confluent, the media was replaced with neuronal induction media [DMEM/F-12 GlutaMAX (Gibco), Neurobasal (Gibco), 0.5x N-2(Gibco), 0.5x B27(Gibco), 0.5x GlutaMAX (Thermo Fisher Scientific), $5 \mu \mathrm{g} / \mathrm{ml}$ insulin (Sigma-Aldrich), 0.5x NEAA (Thermo Fisher Scientific), 100 $\mu \mathrm{M}$ 2-mercaptoethanol (Sigma-Aldrich), 1x Penicillin/Streptomycin (Gibco)] with $1 \mu \mathrm{M}$ Dorsomorphin (Tocris) and $10 \mu \mathrm{M}$ SB431542 (Tocris) for 11 days. Then, cells were transferred to a new Matrigel-coated plate. When the neural rosette is apparent, cells were transferred to the new plate at the density of $5 \times 10^{6}$. Cells were then fed with neuronal induction media containing $20 \mathrm{ng} / \mathrm{ml} \mathrm{FGF2} \mathrm{(Peprotech).}$

\section{Astrocyte differentiation}

hiPSC-derived astrocytes were generated as described previously (TCW et al., 2017). Briefly, NPCs were seeded at $1.5 \times 10^{5}$ cells/well in a 6-well plate. The next day, neuronal induction media were replaced with Astrocyte Media (Sciencell). AM was changed every 2 days. After 4 weeks of differentiation, cells were suspended with TrypLE (Gibco) and sorted with an anti-GLAST-PE antibody (Miltenyl Biotec) using a flow cytometer (Sony SH800). The identity of sorted astrocytes was confirmed by immunocytochemistry with anti-GFAP and anti-AQP4 antibodies. ACM were prepared by culture of astrocytes with 
Neurobasal, 1 x GlutaMAX, 0.5x N-2, 0.5x B-27 (without vitamin A), BSA (Thermo Fisher Scientific).

\section{Neuron differentiation}

Neurons were differentiated from hiPSCs as previously described (Lin et al., 2018). Briefly, hiPSCs were seed on a matrigel-coated 6-well plate at a density of $4 \times 10^{6}$ well with mTeSR1 media containing $20 \mu \mathrm{M}$ ROCK inhibitor Y-27632 (Tocris). After $1.5 \mathrm{hr}$, lentivirus with TetO-NGN2-GFP-puro and rtTA expressing vectors were treated. Next day, media was replaced with neuronal differentiation media [DMEM/F12(Gibco), 1x N2, 1x NEAA] with 10 ng/ml BDNF (Peprotech), 10 ng/ml NT-3 (Peprotech), $0.2 \mu \mathrm{g} / \mathrm{ml}$ laminin (Corning) and $2 \mu \mathrm{g} / \mathrm{ml}$ doxycycline (Sigma-Aldrich)]. Two days later, media was fully changed with media [Neurobasal (Gibco), 1x B27, 1x GlutaMAX] containing $1 \mu \mathrm{g} / \mathrm{ml}$ puromycin (Millipore), $10 \mathrm{ng} / \mathrm{ml} \mathrm{BDNF,} 10 \mathrm{ng} / \mathrm{ml} \mathrm{NT-3,} 0.2 \mu \mathrm{g} / \mathrm{ml}$ laminin and $2 \mu \mathrm{g} / \mathrm{ml}$ doxycycline. After 2 days, cells were transferred to a matrigel-coated $24-$ well plate at a density of $1.5 \times 10^{5} /$ well. The next day, media was replaced with ACM containing $1 \mu \mathrm{M}$ Ara-C (Sigma-Aldrich). Three days later, media were replaced with ACM containing 10 ng/ml BDNF, $10 \mathrm{ng} / \mathrm{ml}$ NT-3, $0.2 \mu \mathrm{g} / \mathrm{ml}$ laminin and $0.5 \mu \mathrm{g} / \mathrm{ml}$ doxycycline. Half volume of media was replaced with fresh media every 4 days, and cells were used for experiments at 5 weeks of differentiation.

\section{Rat primary hippocampal neuron culture}

One day before rat primary hippocampus culture, coverslips were coated with $0.1 \%$ PolyD-Lycine (Sigma-Aldrich). Hippocampi were isolated from embryonic day-18 embryos. 
Isolated hippocampi were collected in HBSS (Thermo), and incubated in papain solution [1 $\mathrm{mM} \mathrm{CaCl}_{2}, 0.5 \mathrm{mM}$ EDTA, Papain (Worthington Biochemical), $\left.1 \mathrm{x} \mathrm{HBSS}\right]$ at $37^{\circ} \mathrm{C}$ for 15 min. After incubation, tissues were washed with HBSS solution and gently agitated to single cells in neuron culture media [Neurobasal media, 1x GlutaMAX, 1x sodium pyruvate (Sigma-Aldrich), 1x B-27, 1x Penicillin-Streptomycin (Gibco), 0.5\% FBS (Gibco). Neurons were used for experiments at DIV 17.

\section{Drug treatment}

Neurons were treated with $20 \mu \mathrm{M}$ cholesterol (Calbiochem) for 4 days or $300 \mu \mathrm{M}$ methyl$\beta$-cyclodextrin (M $\beta C D$, Merck) for 1 day.

\section{Western blotting}

Proteins were extracted from ACM-treated neurons with RIPA buffer (50 mM Tris $\mathrm{pH}$ 8.0, $150 \mathrm{mM} \mathrm{NaCl}, 1 \% \mathrm{NP}-40,0.5 \%$ sodium deoxycholate, $0.1 \%$ SDS). Concentration of proteins was measured by the Bradford assay kit (Bio-Rad). Lysates were subjected to SDS-PAGE and transferred to polyvinylidene difluoride membrane (Bio-Rad), and proved with the indicated antibodies.

\section{Filipin III staining}

Neurons were fixed with 4\% PFA (Biosesang), then cholesterol staining kit (Biovision) was used for filipin III staining. Based on the manufacturer's instruction, neurons were washed with assay buffer, and incubated with filipin staining solution (1:100 diluted in 
assay buffer) for $1 \mathrm{hr}$ at room temperature. Then, cells were washed with assay buffer three times and mounted for imaging.

\section{Immunocytochemistry}

Cells were briefly washed with cold DPBS and fixed with 4\% PFA solution at room temperature for 15 minutes. After fixation, cells were washed with cold DPBS three times and incubated with CTX-B(Invitrogen) for 30 minutes in dark at room temperature. Cells were washed with DPBS three times and permeabilized with $0.1 \%$ Triton-X (Thermo Fisher Scientific) in PBS for 10 min. Then, cells were incubated in blocking buffer $[0.1 \%$ Tween 20 (Promega), 10\% normal donkey serum (Merck), 2\% BSA (Gemini Bio), 1M glycine (Sigma-Aldrich)] for $1 \mathrm{hr}$ at room temperature. Primary antibodies were diluted in blocking buffer, and cells were incubated at $37^{\circ} \mathrm{C}$ for $1 \mathrm{hr}$. After 3 times of washing, secondary antibodies diluted in blocking buffer were treated to cells for $1 \mathrm{hr}$ at room temperature. Washed cells were further incubated with Hoechst 33342 for nucleus staining and mounted for imaging.

\section{Microscopy}

The LSM 800 confocal microscope (Zeiss) was used for imaging, and the Zen software (Zeiss) and Image $\mathrm{J}(\mathrm{NIH})$ were used for data analysis.

\section{ABELISA}

Human $A \beta_{40}$ or $A \beta_{42}$ ELISA detection kit (Thermo Fisher Scientific) was used to detect secreted $A \beta_{40}$ or $A \beta_{42}$ based on the manufacturer's instruction. The absorbance was 
detected with the microplate reader SpectraMax plus 384 (Molecular Devices) at $450 \mathrm{~nm}$ wavelength.

\section{Antibodies}

APP (Abcam), GAPDH (Santa Cruz Biotechnology), CTX-B (Invitrogen), GFAP (Abcam), AQP4 (Merck), Synaptophysin (Sigma-Aldrich), PSD95 (Thermo Fischer Scientific), Hoechst 33342 (Thermo Fischer Scientific), Secondary antibodies (Jackson ImmunoResearch).

\section{Statistical analysis}

The Prism 8 (GraphPad) was used for statistical analysis. Unpaired Student's t-test or oneway ANOVA test with Dunnett's post hoc analysis was used for normal distribution, while the Kruskal-Wallis test was performed for non-normal distribution.

\section{AUTHOR CONTRIBUTIONS}

S.L. and W.J. performed most of the experiments and analyzed data. S.C. and H.L. generated and cultured iPSC-derived astrocytes. Y.J. generated and cultured iPSC-derived neurons. Y.T.L. and L-H.T. provided resources. S.L., W.J., and J.S. conceptualized the project. S.L., W.J., S.C., and J.S. wrote the manuscript. All authors read and approved the final manuscript.

\section{ACKNOWLEDGEMENTS}


We thank all members of the Seo lab for fruitful advice and discussions. This work was supported by the NIH grant (NIA RF1AG062377) to L.-H.T. and the National Research Foundation of Korea (NRF) grants (2018M3C7A1056275, 2019R1C1C1008591) funded by the Ministry of Science, ICT and Future Planning, and the POSCO Science Fellowship of POSCO TJ Park Foundation to J.S.

\section{DECLARATION OF INTERESTS}

The authors declared that they have no competing interests.

\section{REFERENCES}

2020 Alzheimer's disease facts and figures, 2020. 2020 Alzheimer's disease facts and figures. Alzheimers Dement 16, 391-460. doi:10.1002/alz.12068

Beel, A.J., Mobley, C.K., Kim, H.J., Tian, F., Hadziselimovic, A., Jap, B., Prestegard, J.H., Sanders, C.R., 2008. Structural studies of the transmembrane C-terminal domain of the amyloid precursor protein (APP): does APP function as a cholesterol sensor? Biochemistry 47, 9428-9446. doi:10.1021/bi800993c

Bero, A.W., Yan, P., Roh, J.H., Cirrito, J.R., Stewart, F.R., Raichle, M.E., Lee, J.-M., Holtzman, D.M., 2011. Neuronal activity regulates the regional vulnerability to amyloid- $\beta$ deposition. Nat. Neurosci. 14, 750-756. doi:10.1038/nn.2801

$\mathrm{Bu}, \mathrm{G} ., 2009$. Apolipoprotein $\mathrm{E}$ and its receptors in Alzheimer's disease: pathways, pathogenesis and therapy. Nat. Rev. Neurosci. 10, 333-344. doi:10.1038/nrn2620

Canter, R.G., Penney, J., Tsai, L.-H., 2016. The road to restoring neural circuits for the treatment of Alzheimer's disease. Nature 539, 187-196. doi:10.1038/nature20412

Cheng, H., Vetrivel, K.S., Gong, P., Meckler, X., Parent, A., Thinakaran, G., 2007. Mechanisms of disease: new therapeutic strategies for Alzheimer's disease--targeting APP processing in lipid rafts. Nat Clin Pract Neurol 3, 374-382. doi:10.1038/ncpneuro0549

Cho, Y.Y., Kwon, O.-H., Park, M.K., Kim, T.-W., Chung, S., 2019. Elevated cellular cholesterol in Familial Alzheimer's presenilin 1 mutation is associated with lipid raft localization of $\beta$-amyloid precursor protein. PLoS ONE 14, e0210535. doi:10.1371/journal.pone.0210535

Corder, E.H., Saunders, A.M., Strittmatter, W.J., Schmechel, D.E., Gaskell, P.C., Small, G.W., Roses, A.D., Haines, J.L., Pericak-Vance, M.A., 1993. Gene dose of 
apolipoprotein E type 4 allele and the risk of Alzheimer's disease in late onset families. Science 261, 921-923. doi:10.1126/science.8346443

Cossec, J.-C., Simon, A., Marquer, C., Moldrich, R.X., Leterrier, C., Rossier, J.,

Duyckaerts, C., Lenkei, Z., Potier, M.-C., 2010. Clathrin-dependent APP endocytosis and $A \beta$ secretion are highly sensitive to the level of plasma membrane cholesterol.

Biochimica et Biophysica Acta (BBA) - Molecular and Cell Biology of Lipids 1801, 846-852. doi:10.1016/j.bbalip.2010.05.010

Cutler, R.G., Kelly, J., Storie, K., Pedersen, W.A., Tammara, A., Hatanpaa, K., Troncoso, J.C., Mattson, M.P., 2004. Involvement of oxidative stress-induced abnormalities in ceramide and cholesterol metabolism in brain aging and Alzheimer's disease. Proc.

Natl. Acad. Sci. U.S.A. 101, 2070-2075. doi:10.1073/pnas.0305799101

Das, U., Scott, D.A., Ganguly, A., Koo, E.H., Tang, Y., Roy, S., 2013. Activity-Induced Convergenceof APP and BACE-1 in Acidic Microdomains via an EndocytosisDependent Pathway. Neuron 79, 447-460. doi:10.1016/j.neuron.2013.05.035

De Strooper, B., Karran, E., 2016. The Cellular Phase of Alzheimer's Disease. Cell 164, 603-615. doi:10.1016/j.cell.2015.12.056

Djelti, F., Braudeau, J., Hudry, E., Dhenain, M., Varin, J., Bièche, I., Marquer, C., Chali, F., Ayciriex, S., Auzeil, N., Alves, S., Langui, D., Potier, M.-C., Laprevote, O., Vidaud, M., Duyckaerts, C., Miles, R., Aubourg, P., Cartier, N., 2015. CYP46A1 inhibition, brain cholesterol accumulation and neurodegeneration pave the way for Alzheimer's disease. Brain 138, 2383-2398. doi:10.1093/brain/awv166

Fernandez, C.G., Hamby, M.E., McReynolds, M.L., Ray, W.J., 2019. The Role of APOE4 in Disrupting the Homeostatic Functions of Astrocytes and Microglia in Aging and Alzheimer's Disease. Front Aging Neurosci 11, 14. doi:10.3389/fnagi.2019.00014

Hering, H., Lin, C.-C., Sheng, M., 2003. Lipid rafts in the maintenance of synapses, dendritic spines, and surface AMPA receptor stability. J. Neurosci. 23, 3262-3271.

Krasemann, S., Madore, C., Cialic, R., Baufeld, C., Calcagno, N., Fatimy, El, R., Beckers, L., O’Loughlin, E., Xu, Y., Fanek, Z., Greco, D.J., Smith, S.T., Tweet, G., Humulock, Z., Zrzavy, T., Conde-Sanroman, P., Gacias, M., Weng, Z., Chen, H., Tjon, E., Mazaheri, F., Hartmann, K., Madi, A., Ulrich, J.D., Glatzel, M., Worthmann, A., Heeren, J., Budnik, B., Lemere, C., Ikezu, T., Heppner, F.L., Litvak, V., Holtzman, D.M., Lassmann, H., Weiner, H.L., Ochando, J., Haass, C., Butovsky, O., 2017. The TREM2-APOE Pathway Drives the Transcriptional Phenotype of Dysfunctional Microglia in Neurodegenerative Diseases. Immunity 47, 566-581.e9. doi:10.1016/j.immuni.2017.08.008

Kunkle, B.W., Grenier-Boley, B., Sims, R., Bis, J.C., Damotte, V., Naj, A.C., Boland, A., Vronskaya, M., van der Lee, S.J., Amlie-Wolf, A., Bellenguez, C., Frizatti, A., Chouraki, V., Martin, E.R., Sleegers, K., Badarinarayan, N., Jakobsdottir, J., Hamilton-Nelson, K.L., Moreno-Grau, S., Olaso, R., Raybould, R., Chen, Y., 
Kuzma, A.B., Hiltunen, M., Morgan, T., Ahmad, S., Vardarajan, B.N., Epelbaum, J., Hoffmann, P., Boada, M., Beecham, G.W., Garnier, J.-G., Harold, D., Fitzpatrick, A.L., Valladares, O., Moutet, M.-L., Gerrish, A., Smith, A.V., Qu, L., Bacq, D., Denning, N., Jian, X., Zhao, Y., Del Zompo, M., Fox, N.C., Choi, S.-H., Mateo, I., Hughes, J.T., Adams, H.H., Malamon, J., Sanchez-Garcia, F., Patel, Y., Brody, J.A., Dombroski, B.A., Naranjo, M.C.D., Daniilidou, M., Eiriksdottir, G., Mukherjee, S., Wallon, D., Uphill, J., Aspelund, T., Cantwell, L.B., Garzia, F., Galimberti, D., Hofer, E., Butkiewicz, M., Fin, B., Scarpini, E., Sarnowski, C., Bush, W.S., Meslage, S., Kornhuber, J., White, C.C., Song, Y., Barber, R.C., Engelborghs, S., Sordon, S., Voijnovic, D., Adams, P.M., Vandenberghe, R., Mayhaus, M., Cupples, L.A., Albert, M.S., De Deyn, P.P., Gu, W., Himali, J.J., Beekly, D., Squassina, A., Hartmann, A.M., Orellana, A., Blacker, D., Rodriguez-Rodriguez, E., Lovestone, S., Garcia, M.E., Doody, R.S., Munoz-Fernadez, C., Sussams, R., Lin, H., Fairchild, T.J., Benito, Y.A., Holmes, C., Karamujić-Čomić, H., Frosch, M.P., Thonberg, H., Maier, W., Roshchupkin, G., Ghetti, B., Giedraitis, V., Kawalia, A., Li, S., Huebinger, R.M., Kilander, L., Moebus, S., Hernández, I., Kamboh, M.I., Brundin, R., Turton, J., Yang, Q., Katz, M.J., Concari, L., Lord, J., Beiser, A.S., Keene, C.D., Helisalmi, S., Kloszewska, I., Kukull, W.A., Koivisto, A.M., Lynch, A., Tarraga, L., Larson, E.B., Haapasalo, A., Lawlor, B., Mosley, T.H., Lipton, R.B., Solfrizzi, V., Gill, M., Longstreth, W.T., Montine, T.J., Frisardi, V., Diez-Fairen, M., Rivadeneira, F., Petersen, R.C., Deramecourt, V., Alvarez, I., Salani, F., Ciaramella, A., Boerwinkle, E., Reiman, E.M., Fievet, N., Rotter, J.I., Reisch, J.S., Hanon, O., Cupidi, C., Andre Uitterlinden, A.G., Royall, D.R., Dufouil, C., Maletta, R.G., de Rojas, I., Sano, M., Brice, A., Cecchetti, R., George-Hyslop, P.S., Ritchie, K., Tsolaki, M., Tsuang, D.W., Dubois, B., Craig, D., Wu, C.-K., Soininen, H., Avramidou, D., Albin, R.L., Fratiglioni, L., Germanou, A., Apostolova, L.G., Keller, L., Koutroumani, M., Arnold, S.E., Panza, F., Gkatzima, O., Asthana, S., Hannequin, D., Whitehead, P., Atwood, C.S., Caffarra, P., Hampel, H., Quintela, I., Carracedo, Á., Lannfelt, L., Rubinsztein, D.C., Barnes, L.L., Pasquier, F., Frölich, L., Barral, S., McGuinness, B., Beach, T.G., Johnston, J.A., Becker, J.T., Passmore, P., Bigio, E.H., Schott, J.M., Bird, T.D., Warren, J.D., Boeve, B.F., Lupton, M.K., Bowen, J.D., Proitsi, P., Boxer, A., Powell, J.F., Burke, J.R., Kauwe, J.S.K., Burns, J.M., Mancuso, M., Buxbaum, J.D., Bonuccelli, U., Cairns, N.J., McQuillin, A., Cao, C., Livingston, G., Carlson, C.S., Bass, N.J., Carlsson, C.M., Hardy, J., Carney, R.M., Brás, J., Carrasquillo, M.M., Guerreiro, R., Allen, M., Chui, H.C., Fisher, E., Masullo, C., Crocco, E.A., DeCarli, C., Bisceglio, G., Dick, M., Ma, L., Duara, R., Graff-Radford, N.R., Evans, D.A., Hodges, A., Faber, K.M., Scherer, M., Fallon, K.B., Riemenschneider, M., Fardo, D.W., Heun, R., Farlow, M.R., Kölsch, H., Ferris, S., Leber, M., Foroud, T.M., Heuser, I., Galasko, D.R., Giegling, I., Gearing, M., Hüll, M., Geschwind, D.H., Gilbert, J.R., Morris, J., Green, R.C., Mayo, K., Growdon, J.H., Feulner, T., 
Hamilton, R.L., Harrell, L.E., Drichel, D., Honig, L.S., Cushion, T.D., Huentelman, M.J., Hollingworth, P., Hulette, C.M., Hyman, B.T., Marshall, R., Jarvik, G.P., Meggy, A., Abner, E., Menzies, G.E., Jin, L.-W., Leonenko, G., Real, L.M., Jun, G.R., Baldwin, C.T., Grozeva, D., Karydas, A., Russo, G., Kaye, J.A., Kim, R., Jessen, F., Kowall, N.W., Vellas, B., Kramer, J.H., Vardy, E., LaFerla, F.M., Jöckel, K.-H., Lah, J.J., Dichgans, M., Leverenz, J.B., Mann, D., Levey, A.I., PickeringBrown, S., Lieberman, A.P., Klopp, N., Lunetta, K.L., Wichmann, H.-E., Lyketsos, C.G., Morgan, K., Marson, D.C., Brown, K., Martiniuk, F., Medway, C., Mash, D.C., Nöthen, M.M., Masliah, E., Hooper, N.M., McCormick, W.C., Daniele, A., McCurry, S.M., Bayer, A., McDavid, A.N., Gallacher, J., McKee, A.C., van den Bussche, H., Mesulam, M., Brayne, C., Miller, B.L., Riedel-Heller, S., Miller, C.A., Miller, J.W., Al-Chalabi, A., Morris, J.C., Shaw, C.E., Myers, A.J., Wiltfang, J., O'Bryant, S., Olichney, J.M., Alvarez, V., Parisi, J.E., Singleton, A.B., Paulson, H.L., Collinge, J., Perry, W.R., Mead, S., Peskind, E., Cribbs, D.H., Rossor, M., Pierce, A., Ryan, N.S., Poon, W.W., Nacmias, B., Potter, H., Sorbi, S., Quinn, J.F., Sacchinelli, E., Raj, A., Spalletta, G., Raskind, M., Caltagirone, C., Bossù, P., Orfei, M.D., Reisberg, B., Clarke, R., Reitz, C., Smith, A.D., Ringman, J.M., Warden, D., Roberson, E.D., Wilcock, G., Rogaeva, E., Bruni, A.C., Rosen, H.J., Gallo, M., Rosenberg, R.N., Ben-Shlomo, Y., Sager, M.A., Mecocci, P., Saykin, A.J., Pastor, P., Cuccaro, M.L., Vance, J.M., Schneider, J.A., Schneider, L.S., Slifer, S., Seeley, W.W., Smith, A.G., Sonnen, J.A., Spina, S., Stern, R.A., Swerdlow, R.H., Tang, M., Tanzi, R.E., Trojanowski, J.Q., Troncoso, J.C., Van Deerlin, V.M., Van Eldik, L.J., Vinters, H.V., Vonsattel, J.P., Weintraub, S., Welsh-Bohmer, K.A., Wilhelmsen, K.C., Williamson, J., Wingo, T.S., Woltjer, R.L., Wright, C.B., Yu, C.-E., Yu, L., Saba, Y., Pilotto, A., Bullido, M.J., Peters, O., Crane, P.K., Bennett, D., Bosco, P., Coto, E., Boccardi, V., De Jager, P.L., Lleo, A., Warner, N., Lopez, O.L., Ingelsson, M., Deloukas, P., Cruchaga, C., Graff, C., Gwilliam, R., Fornage, M., Goate, A.M., Sanchez-Juan, P., Kehoe, P.G., Amin, N., Ertekin-Taner, N., Berr, C., Debette, S., Love, S., Launer, L.J., Younkin, S.G., Dartigues, J.-F., Corcoran, C., Ikram, M.A., Dickson, D.W., Nicolas, G., Campion, D., Tschanz, J., Schmidt, H., Hakonarson, H., Clarimon, J., Munger, R., Schmidt, R., Farrer, L.A., Van Broeckhoven, C., C O'Donovan, M., DeStefano, A.L., Jones, L., Haines, J.L., Deleuze, J.-F., Owen, M.J., Gudnason, V., Mayeux, R., Escott-Price, V., Psaty, B.M., Ramirez, A., Wang, L.-S., Ruiz, A., van Duijn, C.M., Holmans, P.A., Seshadri, S., Williams, J., Amouyel, P., Schellenberg, G.D., Lambert, J.-C., Pericak-Vance, M.A., Alzheimer Disease Genetics Consortium (ADGC), European Alzheimer's Disease Initiative (EADI), Cohorts for Heart and Aging Research in Genomic Epidemiology Consortium (CHARGE), Genetic and Environmental Risk in AD/Defining Genetic, Polygenic and Environmental Risk for Alzheimer's Disease Consortium (GERAD/PERADES), 2019. Genetic meta-analysis of diagnosed Alzheimer's disease identifies new risk loci 
and implicates $A \beta$, tau, immunity and lipid processing. Nat. Genet. 51, 414-430. doi:10.1038/s41588-019-0358-2

Lazar, A.N., Bich, C., Panchal, M., Desbenoit, N., Petit, V.W., Touboul, D., Dauphinot, L., Marquer, C., Laprevote, O., Brunelle, A., Duyckaerts, C., 2013. Time-of-flight secondary ion mass spectrometry (TOF-SIMS) imaging reveals cholesterol overload in the cerebral cortex of Alzheimer disease patients. Acta Neuropathol. 125, 133144. doi:10.1007/s00401-012-1041-1

Lin, Y.-T., Seo, J., Gao, F., Feldman, H.M., Wen, H.-L., Penney, J., Cam, H.P., Gjoneska, E., Raja, W.K., Cheng, J., Rueda, R., Kritskiy, O., Abdurrob, F., Peng, Z., Milo, B., Yu, C.J., Elmsaouri, S., Dey, D., Ko, T., Yankner, B.A., Tsai, L.-H., 2018. APOE4 Causes Widespread Molecular and Cellular Alterations Associated with Alzheimer's Disease Phenotypes in Human iPSC-Derived Brain Cell Types. Neuron 98, 1141-1154.e7. doi:10.1016/j.neuron.2018.05.008

Liu, C.-C., Liu, C.-C., Kanekiyo, T., Xu, H., Bu, G., 2013. Apolipoprotein E and Alzheimer disease: risk, mechanisms and therapy. Nat Rev Neurol 9, 106-118. doi:10.1038/nrneurol.2012.263

Liu, C.-C., Zhao, N., Fu, Y., Wang, N., Linares, C., Tsai, C.-W., Bu, G., 2017. ApoE4 Accelerates Early Seeding of Amyloid Pathology. Neuron 96, 1024-1032.e3. doi:10.1016/j.neuron.2017.11.013

Marquer, C., Devauges, V., Cossec, J.-C., Liot, G., Lécart, S., Saudou, F., Duyckaerts, C., Lévêque-Fort, S., Potier, M.-C., 2011. Local cholesterol increase triggers amyloid precursor protein-Bacel clustering in lipid rafts and rapid endocytosis. FASEB J. 25, 1295-1305. doi:10.1096/fj.10-168633

Marquer, C., Laine, J., Dauphinot, L., Hanbouch, L., Lemercier-Neuillet, C., Pierrot, N., Bossers, K., Le, M., Corlier, F., Benstaali, C., Saudou, F., Thinakaran, G., Cartier, N., Octave, J.-N., Duyckaerts, C., Potier, M.-C., 2014. Increasing membrane cholesterol of neurons in culture recapitulates Alzheimer's disease early phenotypes. Mol Neurodegener 9, 1-13. doi:10.1186/1750-1326-9-60

Mauch, D.H., Nägler, K., Schumacher, S., Göritz, C., Müller, E.C., Otto, A., Pfrieger, F.W., 2001. CNS synaptogenesis promoted by glia-derived cholesterol. Science 294, 1354-1357. doi:10.1126/science.294.5545.1354

Meyer, K., Feldman, H.M., Lu, T., Drake, D., Lim, E.T., Ling, K.-H., Bishop, N.A., Pan, Y., Seo, J., Lin, Y.-T., Su, S.C., Church, G.M., Tsai, L.-H., Yankner, B.A., 2019. REST and Neural Gene Network Dysregulation in iPSC Models of Alzheimer's Disease. Cell Rep 26, 1112-1127.e9. doi:10.1016/j.celrep.2019.01.023

Narayan, P., Sienski, G., Bonner, J.M., Lin, Y.-T., Seo, J., Baru, V., Haque, A., Milo, B., Akay, L.A., Graziosi, A., Freyzon, Y., Landgraf, D., Hesse, W.R., Valastyan, J., Barrasa, M.I., Tsai, L.-H., Lindquist, S., 2020. PICALM Rescues Endocytic Defects Caused by the Alzheimer's Disease Risk Factor APOE4. Cell Rep 33, 108224. doi:10.1016/j.celrep.2020.108224 
O'Brien, R.J., Wong, P.C., 2011. Amyloid precursor protein processing and Alzheimer's disease. Annu. Rev. Neurosci. 34, 185-204. doi:10.1146/annurev-neuro-061010113613

Raffai, R.L., Weisgraber, K.H., 2003. Cholesterol: from heart attacks to Alzheimer's disease. J. Lipid Res. 44, 1423-1430. doi:10.1194/j1r.R300007-JLR200

TCW, J., Liang, S.A., Qian, L., Pipalia, N.H., Chao, M.J., Shi, Y., Bertelsen, S.E., Kapoor, M., Marcora, E., Sikora, E., Holtzman, D.M., Maxfield, F.R., Bin Zhang, Wang, M., Poon, W.W., Goate, A.M., 2019. Cholesterol and matrisome pathways dysregulated in human APOE $\varepsilon 4$ glia. bioRxiv 99, 713362. doi:10.1101/713362

TCW, J., Wang, M., Pimenova, A.A., Bowles, K.R., Hartley, B.J., Lacin, E., Machlovi, S.I., Abdelaal, R., Karch, C.M., Phatnani, H., Slesinger, P.A., Zhang, B., Goate, A.M., Brennand, K.J., 2017. An Efficient Platform for Astrocyte Differentiation from Human Induced Pluripotent Stem Cells. Stem Cell Reports 9, 600-614. doi:10.1016/j.stemcr.2017.06.018

Vance, J.E., 2012. Dysregulation of cholesterol balance in the brain: contribution to neurodegenerative diseases. Dis Model Mech 5, 746-755. doi:10.1242/dmm.010124

Wang, C., Najm, R., Xu, Q., Jeong, D.-E., Walker, D., Balestra, M.E., Yoon, S.Y., Yuan, H., Li, G., Miller, Z.A., Miller, B.L., Malloy, M.J., Huang, Y., 2018. Gain of toxic apolipoprotein E4 effects in human iPSC-derived neurons is ameliorated by a smallmolecule structure corrector. Nat. Med. 24, 647-657. doi:10.1038/s41591-018-0004$\mathrm{Z}$

Yamazaki, T., Chang, T.Y., Haass, C., Ihara, Y., 2001. Accumulation and aggregation of amyloid beta-protein in late endosomes of Niemann-pick type $\mathrm{C}$ cells. J. Biol. Chem. 276, 4454-4460. doi:10.1074/jbc.M009598200

Zhang, Y., Pak, C., Han, Y., Ahlenius, H., Zhang, Z., Chanda, S., Marro, S., Patzke, C., Acuna, C., Covy, J., Xu, W., Yang, N., Danko, T., Chen, L., Wernig, M., Südhof, T.C., 2013. Rapid single-step induction of functional neurons from human pluripotent stem cells. Neuron 78, 785-798. doi:10.1016/j.neuron.2013.05.029

\section{FIGURE LEGENDS}

Figure. 1

\section{ApoE4 astrocyte conditioned media increase APP expression and A $\beta$ secretion in} hiPSC-derived neurons.

(A) Schematics for generating astrocytes and excitatory neurons from hiPSCs. (B) Experimental procedure to examine the effect of ApoE3 or ApoE4 ACM treatment on 
hiPSC-derived neurons. (C) Representative images of APP staining from ACM-treated neurons. Scale bar $=10 \mu \mathrm{m}$. (D) Quantification of APP area (left), intensity (middle), and total levels (right; area $\mathrm{x}$ intensity) in ACM-treated neurons. (E) Western blotting for fulllength APP (APP-FL) in ApoE3 or ApoE4 ACM-treated neurons. (Right) levels of APP were normalized to GAPDH expression and quantified. (F) Levels of secreted $A \beta_{40}$ and $\mathrm{A} \beta_{42}$ from $\mathrm{ACM}$-treated neurons were detected by ELISA. ${ }^{*} p<0.05,{ }^{* *} p<0.01$, $* * * p<0.001$ (Student's t-test).

\section{Figure.2}

Cholesterol positively regulates the formation of lipid rafts and its association with APP in hiPSC-derived neurons

(A) Experimental procedure to examine the effect of cholesterol or $\mathrm{M} \beta \mathrm{CD}$ treatment on hiPSC-derived neurons. (B) Representative images of Filipin III staining from cholesterol or M $\beta C D$-treated neurons. Scale bar $=10 \mu \mathrm{m}$. (C) Quantification of Filipin III area (left), intensity (middle), and total levels (right; area x intensity) in cholesterol or M $\beta C D$-treated neurons. (D) Representative images of CTX-B and APP staining from cholesterol or MBCD-treated neurons. Scale bar $=10 \mu \mathrm{m}$. (E) Quantification of CTX-B area (left), intensity (middle), and total levels (right; area x intensity) in cholesterol or M $\beta C D$-treated neurons. (F) Quantification of APP area (left), intensity (middle), and total levels (right; area $\mathrm{x}$ intensity) in cholesterol or MBCD-treated neurons. (G) Quantification of size and APP intensity in CTX-B/APP co-localization area. ${ }^{*} p<0.05,{ }^{* *} p<0.01,{ }^{* * *} p<0.001$, **** $p<0.0001$ (ANOVA test followed by Dunnett's post hoc analysis, or Kruskal-Wallis test). 


\section{Figure.3}

\section{ApoE4 ACM increases neuronal lipid rafts}

(A) Measurement of secretory levels of cholesterols in ApoE3 or ApoE4 ACM via Filipin III intensity in the media. (B) Representative images of Filipin III staining from ApoE3 or ApoE4 ACM-treated neurons. Scale bar $=10 \mu \mathrm{m}$. (C) Quantification of Filipin III area (left), intensity (middle), and total levels (right; area x intensity) in ApoE3 or ApoE4 ACMtreated neurons. (D) Representative images of CTX-B staining from ApoE3 or ApoE4 ACM-treated neurons. Scale bar $=10 \mu \mathrm{m}$. (E) Quantification of CTX-B area (left), intensity (middle), and total levels (right; area x intensity) in ApoE3 or ApoE4 ACM-treated neurons. (F) Experimental procedure to examine the effect of cholesterol reduction in ApoE4 ACM on hiPSC-derived neurons. (G) Representative images of Filipin III staining from ApoE3 ACM, ApoE4 ACM, or ApoE4 ACM with M $\beta C D$-treated neurons. Scale bar $=10 \mu \mathrm{m} .(\mathrm{H})$ Quantification of Filipin III area (left), intensity (middle), and total levels (right; area x intensity) in ApoE3 ACM, ApoE4 ACM, or ApoE4 ACM with M $\beta C D$-treated neurons. (I) Representative images of CTX-B staining from ApoE3 ACM, ApoE4 ACM or ApoE4 ACM with M $\beta C D$-treated neurons. Scale bar $=10 \mu \mathrm{m} .(\mathrm{J})$ Quantification of CTX-B area

(left), intensity (middle), and total levels (right; area $\mathrm{x}$ intensity). ${ }^{*} p<0.05,{ }^{*} p<0.01$, $* * * * p<0.0001$ (Student's t-test, ANOVA test followed by Tukey's post hoc analysis, or Kruskal-Wallis test).

\section{Figure.4}

ApoE4 ACM increases neuronal APP expression and A $\beta$ secretion 
(A) Experimental procedure to examine the effect of cholesterol reduction in ApoE4 ACM on hiPSC-derived neurons. (B) Representative images of APP staining from ApoE3 ACM, ApoE4 ACM, or ApoE4 ACM with MBCD-treated neurons. Scale bar $=10 \mu \mathrm{m}$. (C) Quantification of CTX-B area (left), intensity (middle), and total levels (right; area x intensity). (D) Quantification of APP area (left), intensity (middle), and total levels (right; area $\mathrm{x}$ intensity). (E) Western blotting for full-length APP (APP-FL) in ApoE3 ACM, ApoE4 ACM, or ApoE4 ACM with MßCD-treated neurons. (Right) levels of APP were normalized to GAPDH expression and quantified. (F) Levels of secreted $A \beta_{40}$ and $A \beta_{42}$ from ACM-treated neurons were detected by ELISA. ${ }^{*} p<0.05,{ }^{* *} p<0.01,{ }^{* * *} p<0.001$, ${ }^{* * * *} p<0.0001$ (Student's t-test, ANOVA test followed by Dunnett's post hoc analysis, or Kruskal-Wallis test). 
Human iPSC-derived astrocytes

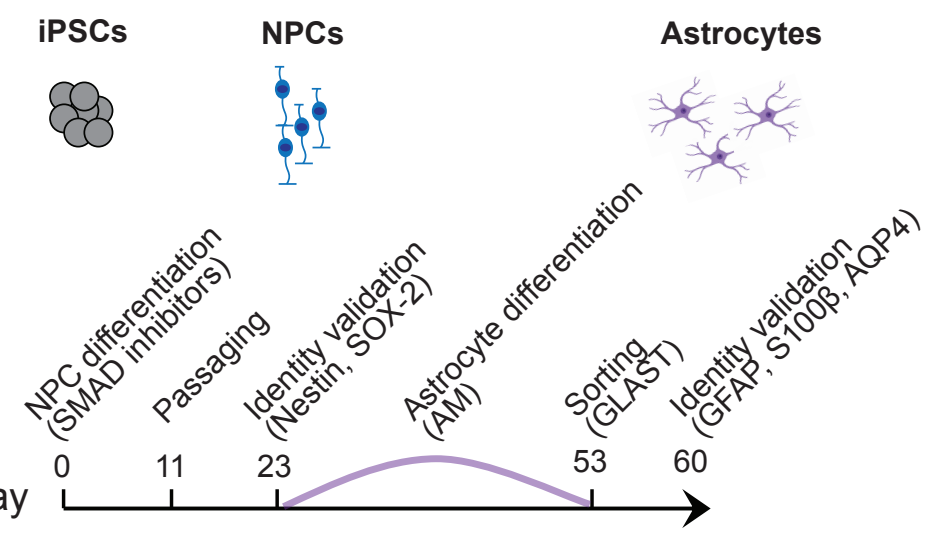

B

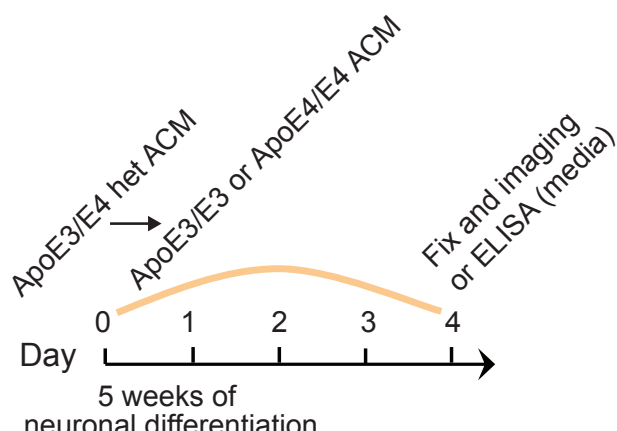

neuronal differentiation

E

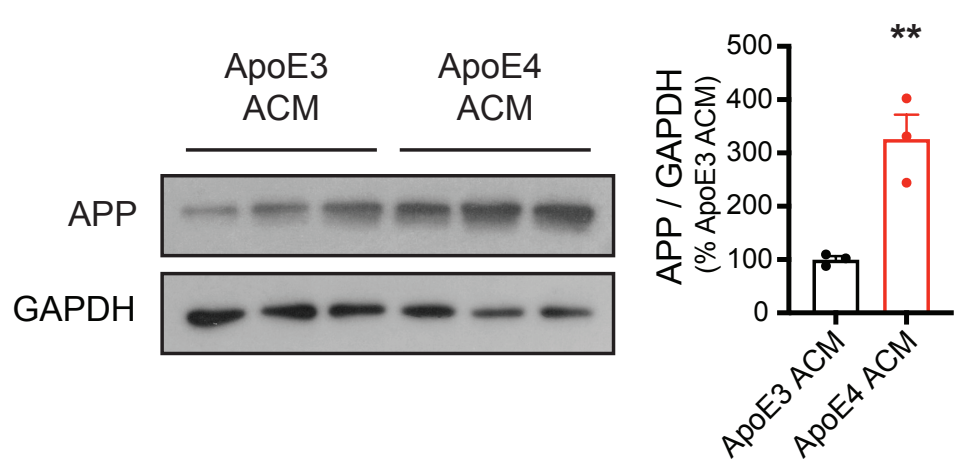

Human iPSC-derived neurons

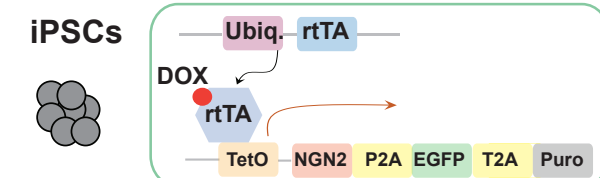

Excitatory Neurons
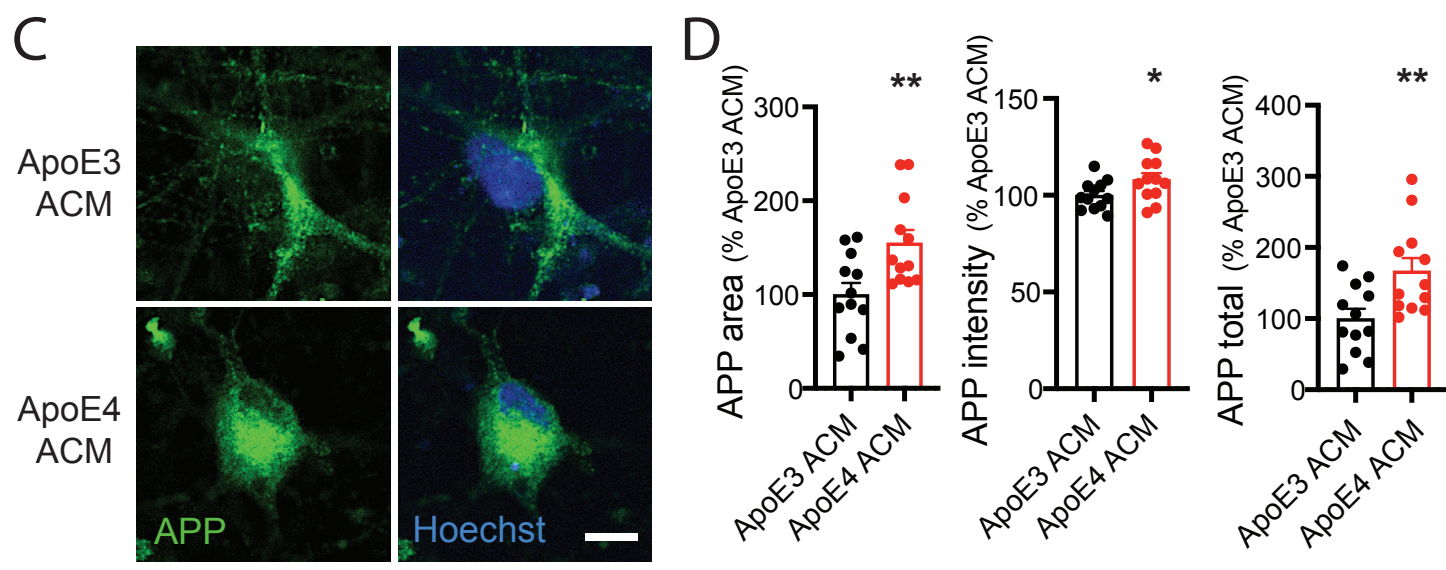

F
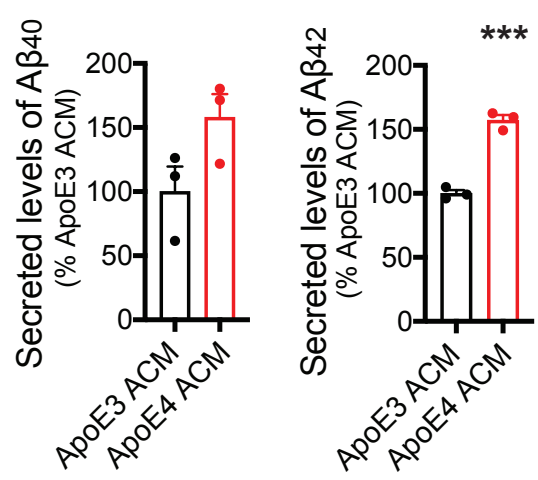
A

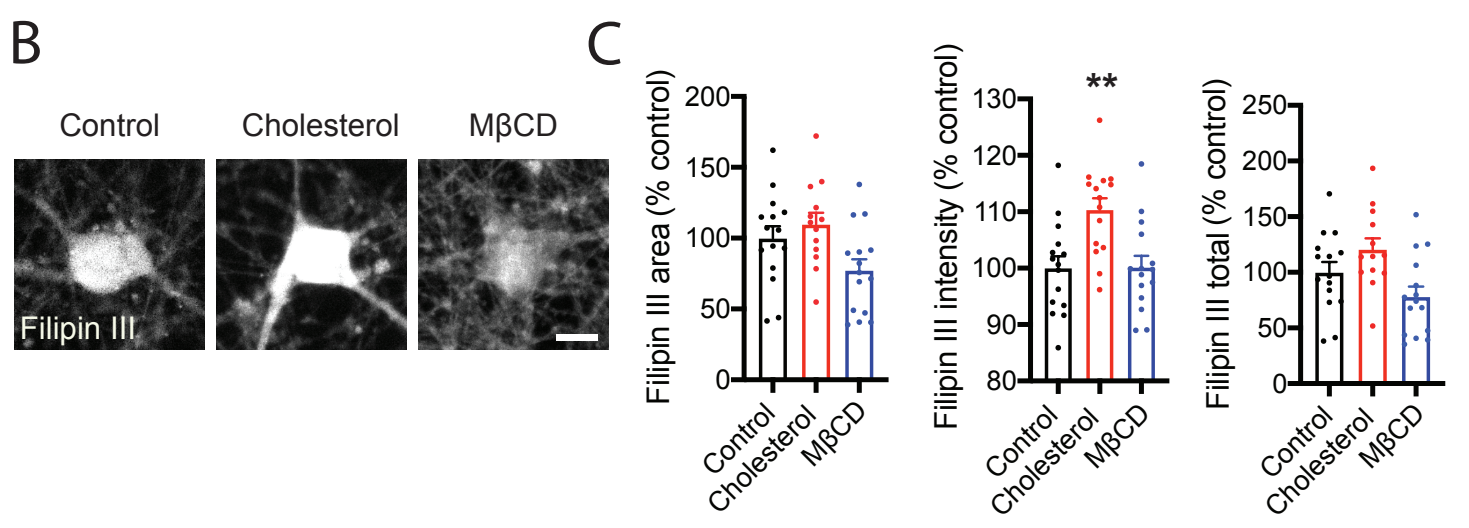

D
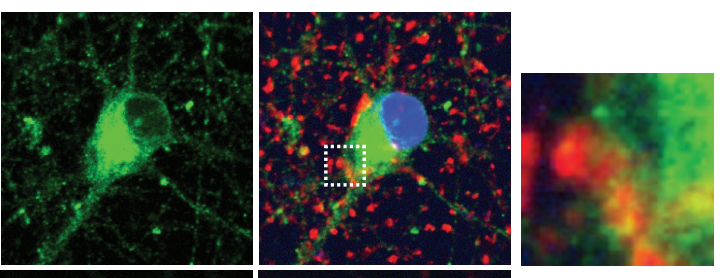

Cholesterot
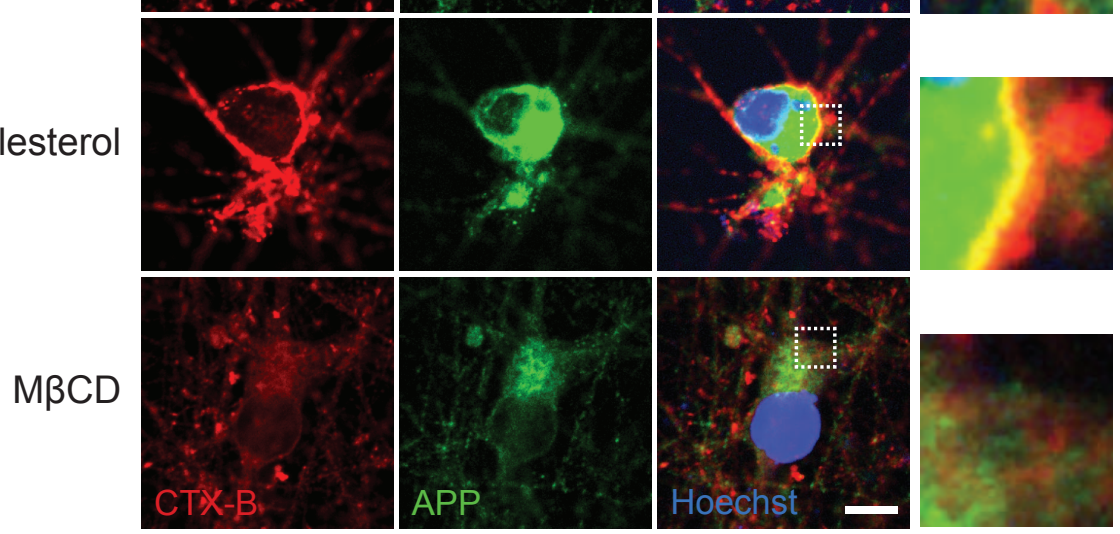

E
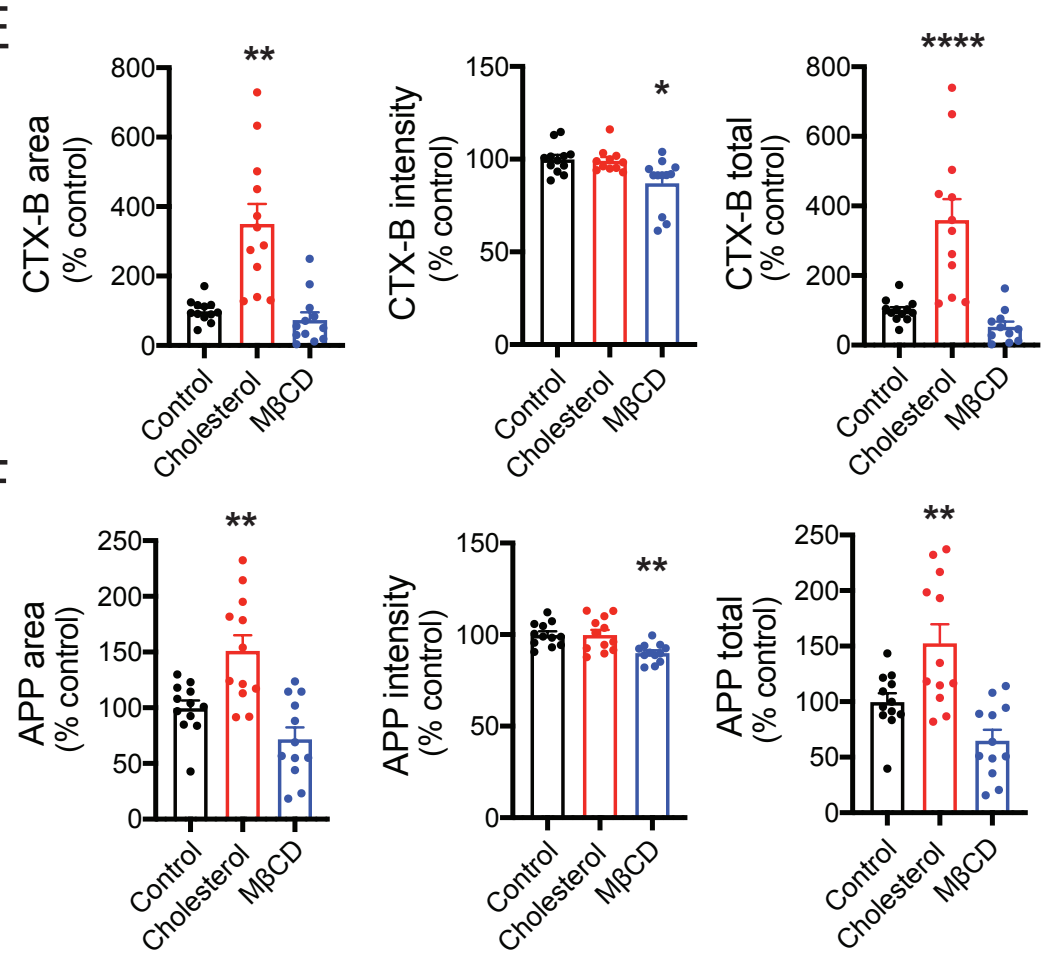

G
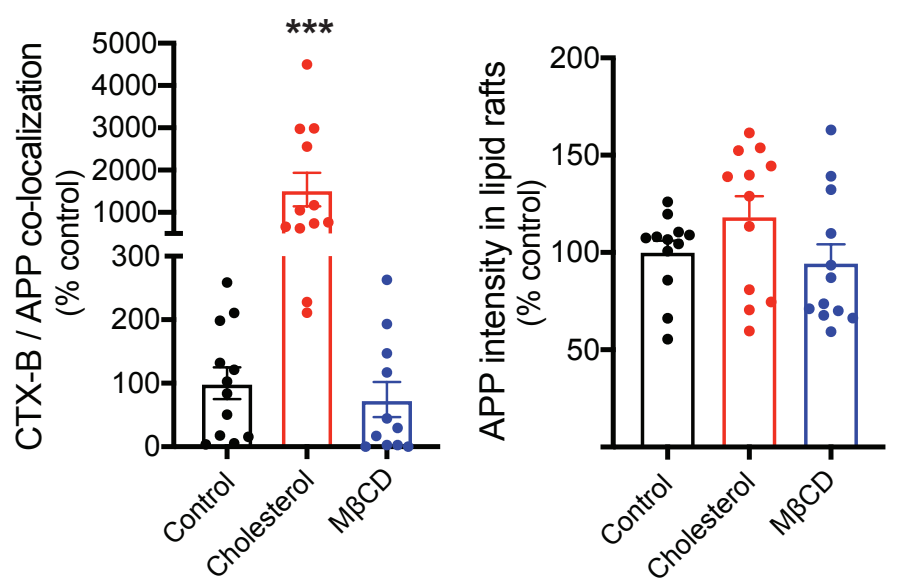

Figure 2 

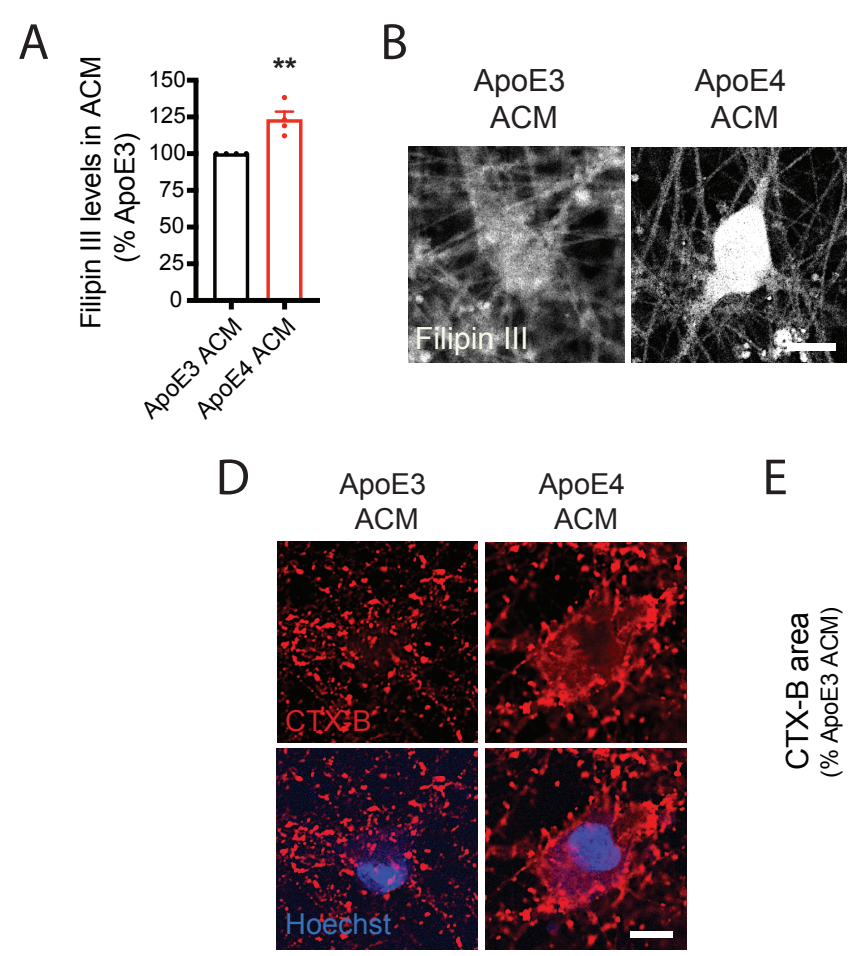

ApoE4

ACM

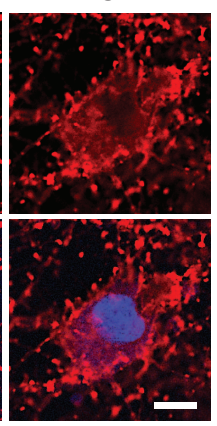

G

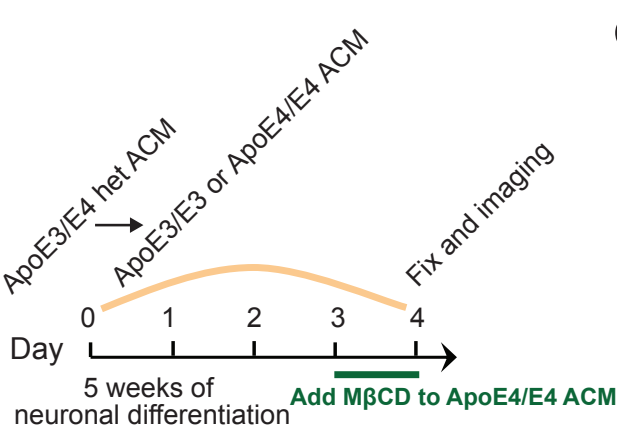

E

neuronal differentiation $A d d \mathrm{M} \beta C D$ to ApoE4/E4 ACM
C

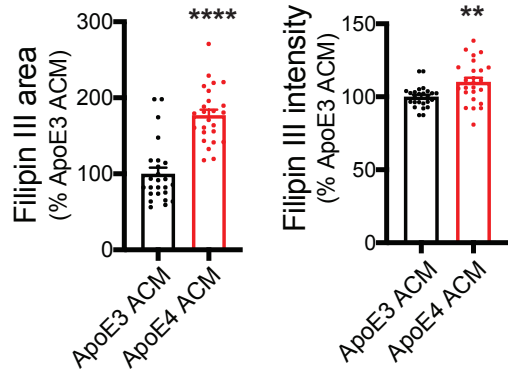

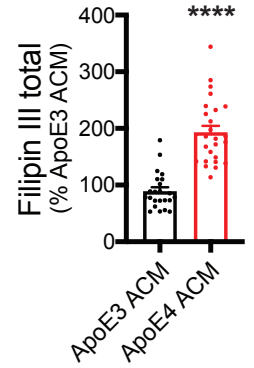
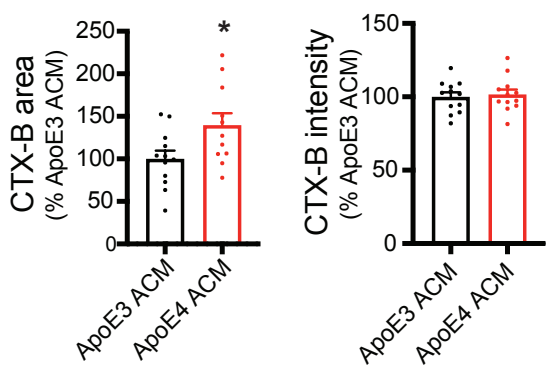

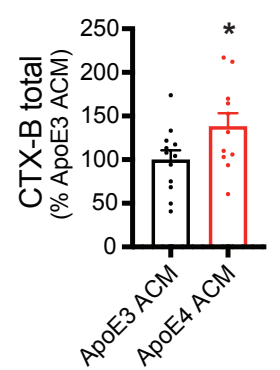

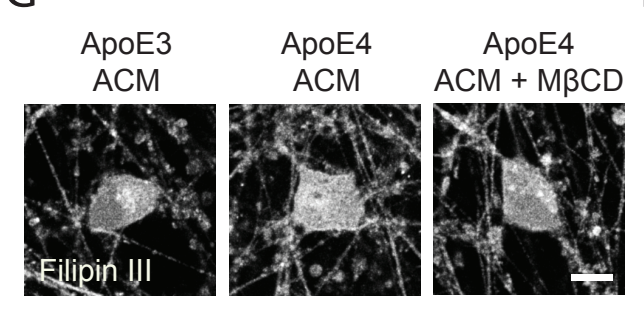

$\mathrm{H}$
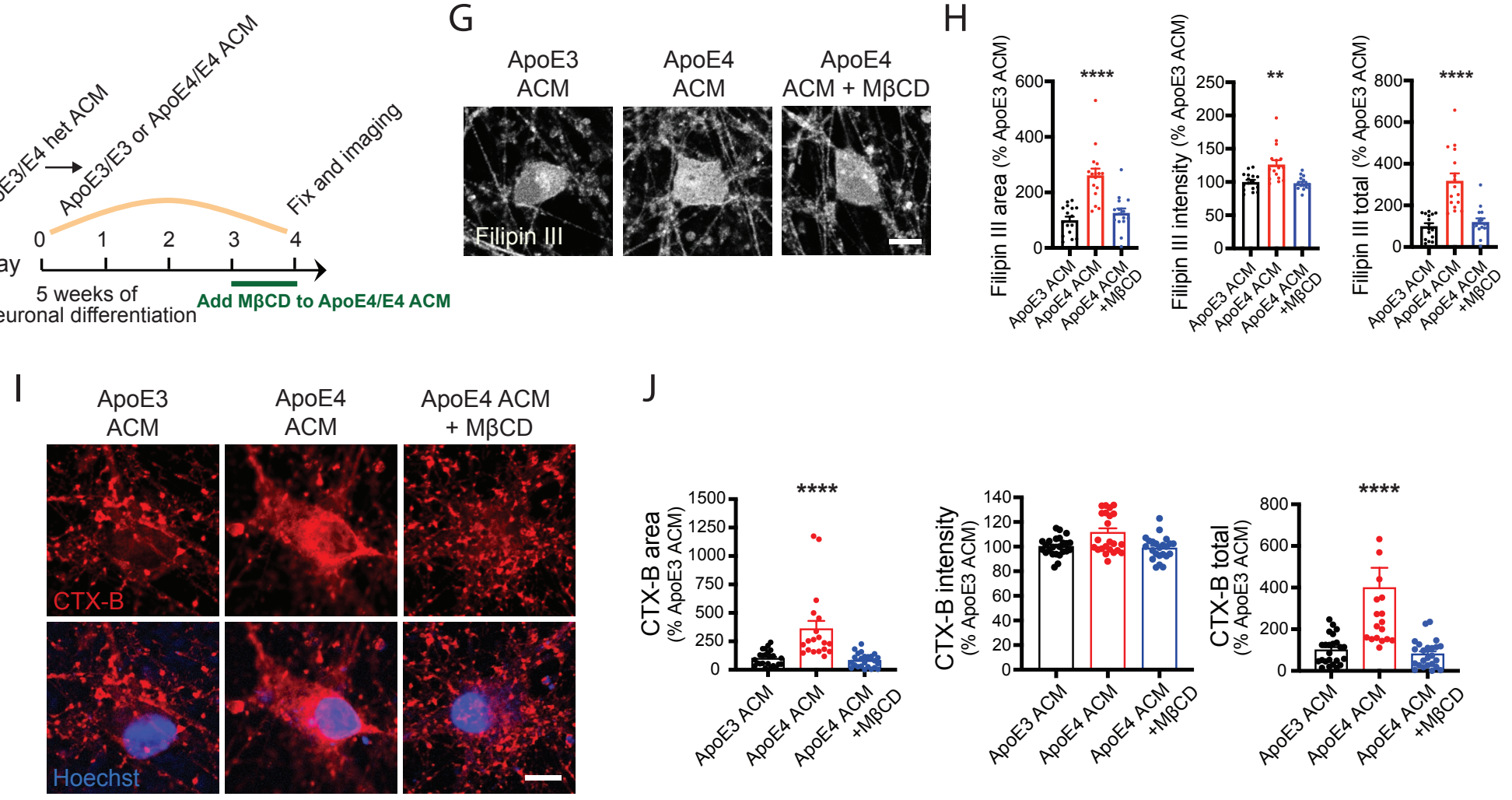

Figure 3 
A

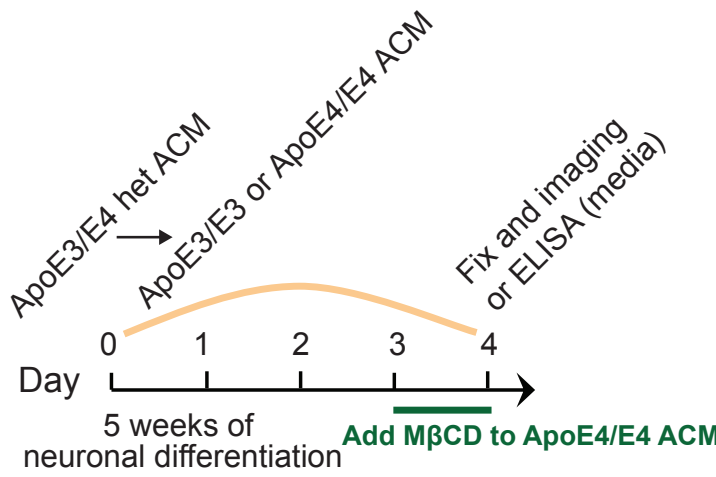

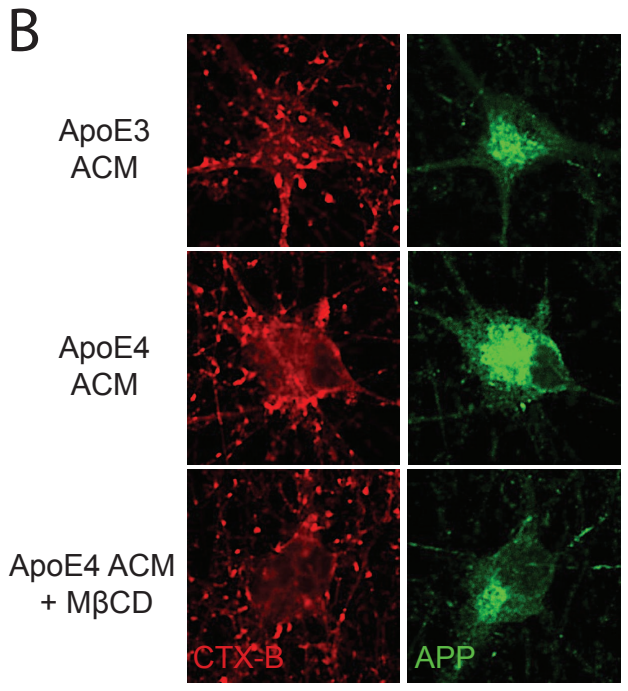

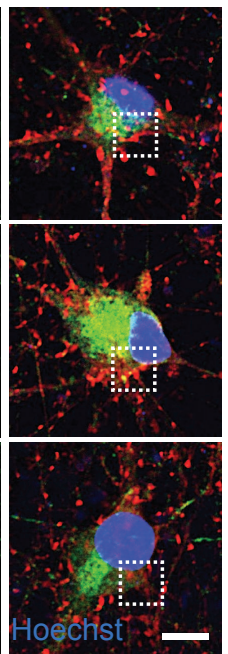

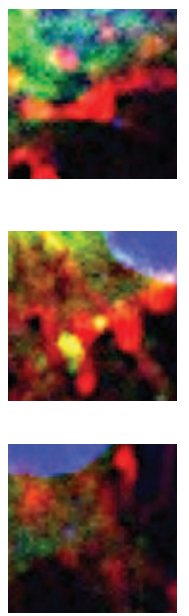

D

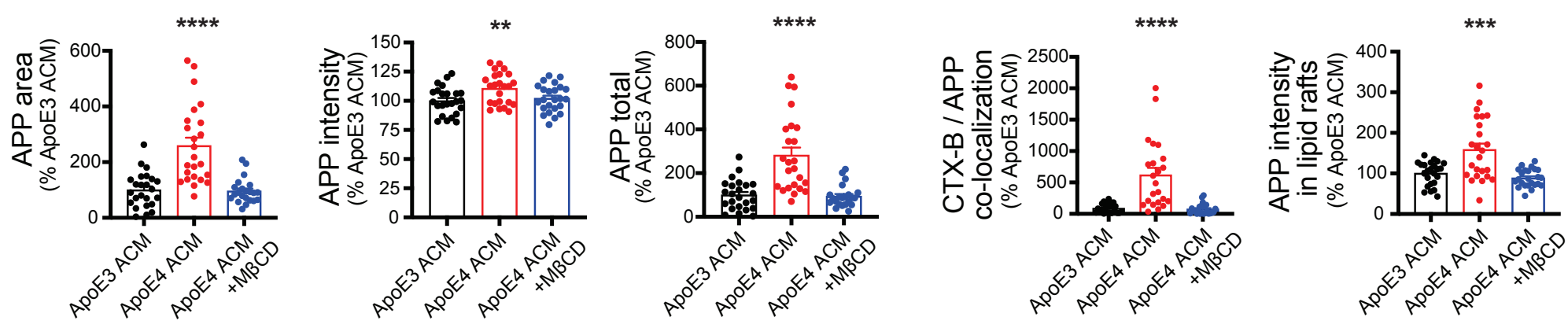

E

F
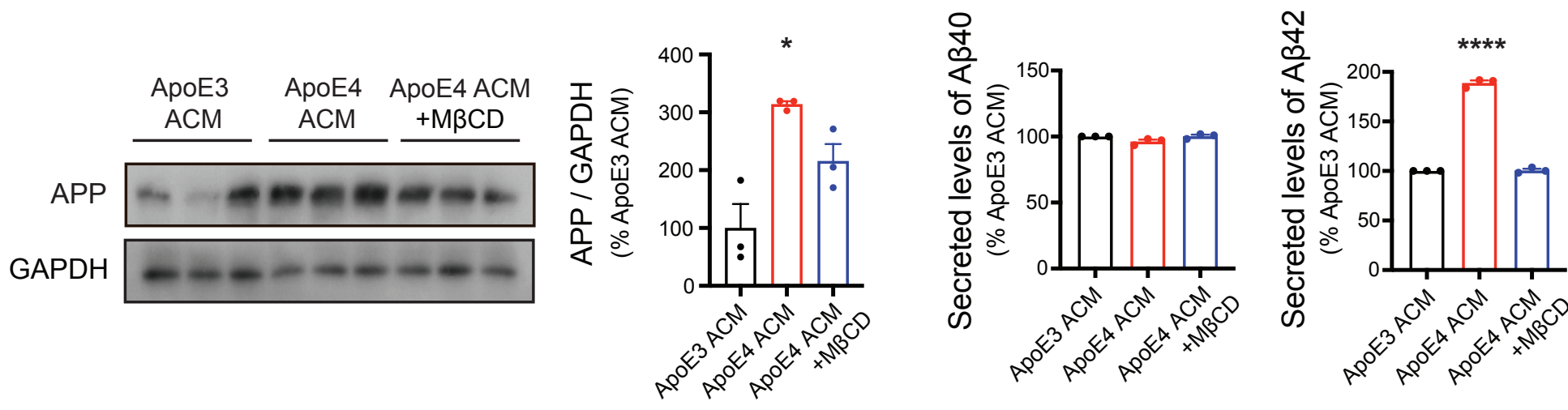


\section{Supplementary Information}

ApoE4-carrying human astrocytes oversupply cholesterol into neurons and promote A $\beta$ generation

Se-In Lee, Woojin Jeong, Sukhee Cho, Hyein Lee, Yonghee Jang, Yuan-Ta Lin, Li-Huei

Tsai and Jinsoo Seo 
Supplementary Figure 1.

A

Human iPSC-derived astrocytes
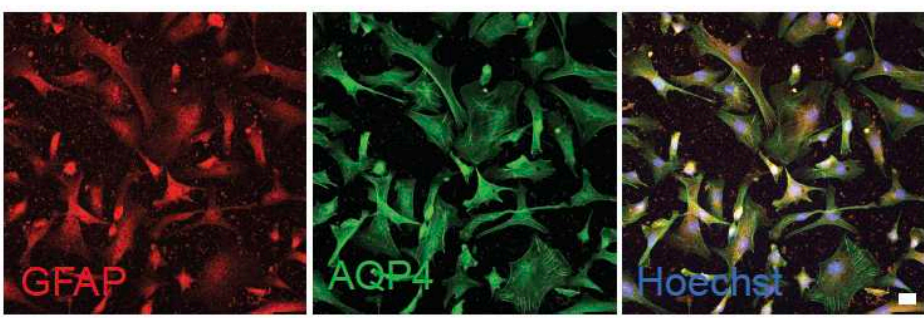

B

Human iPSC-derived excitatory neurons
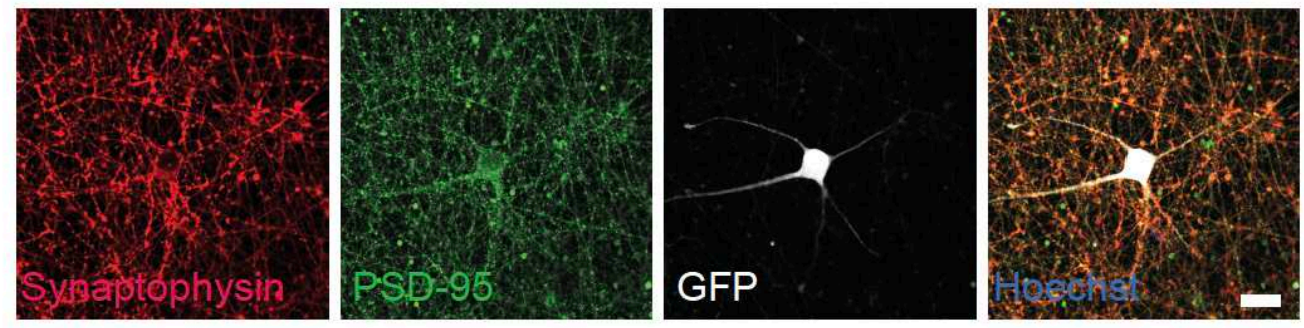


\section{Supplementary Figure 1.}

Validating identity of hiPSC-derived astrocytes and neurons. Related to Figure 1.

(A) Representative images of stained hiPSC-derived astrocytes with astrocytic markers

GFAP and AQP4. Scale bar $=10 \mu \mathrm{m}$. (B) Representative images of stained hiPSC-derived neurons with synaptic markers synaptophysin and PSD-95. Scale bar $=10 \mu \mathrm{m}$. 


\section{Supplementary Figure 2.}
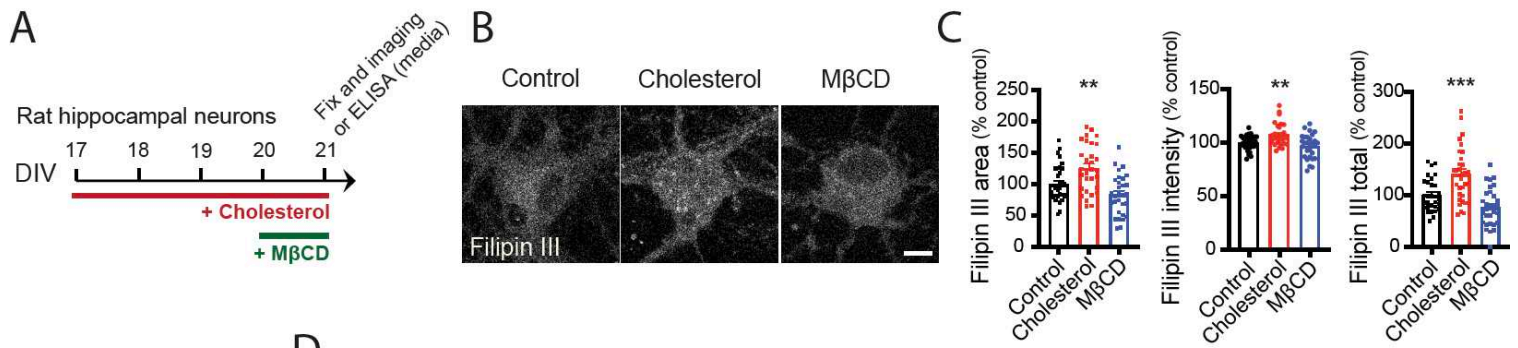

\section{D \\ Dontrol}
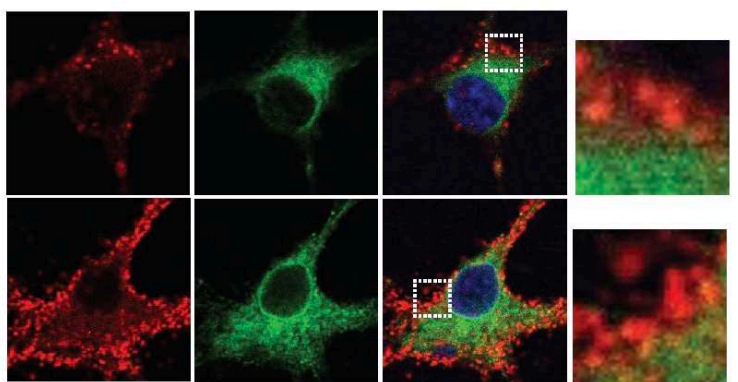

Cholestero

$M \beta C D$
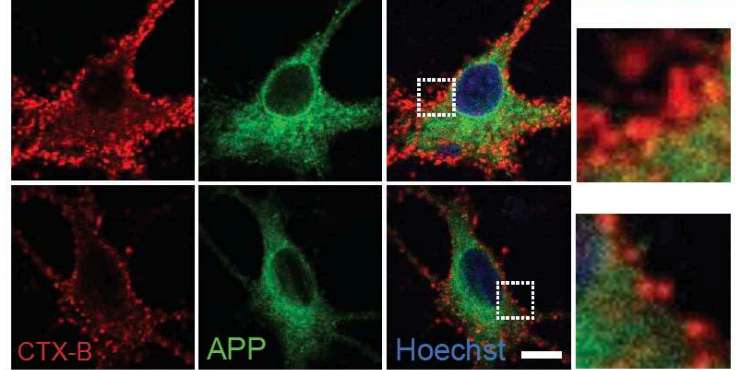

E
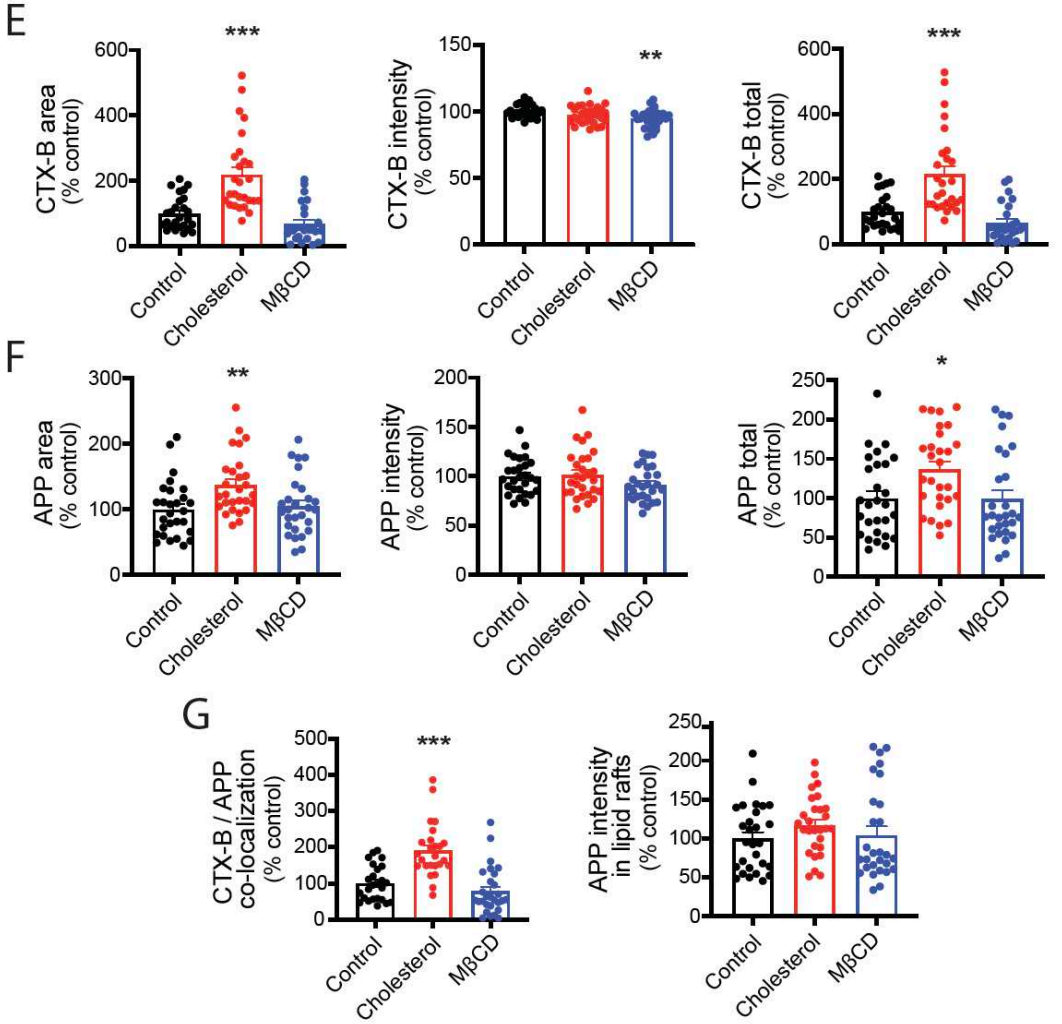


\section{Supplementary Figure 2.}

Cholesterol positively regulates the formation of lipid rafts and its association with APP in rat primary neurons. Related to Figure 2.

(A) Experimental procedure to examine the effect of cholesterol or M $\beta C D$ treatment on rat primary neurons. (B) Representative images of Filipin III staining from cholesterol or M $\beta C D$-treated neurons. Scale bar $=10 \mu \mathrm{m}$. (C) Quantification of Filipin III area (left), intensity (middle), and total levels (right; area x intensity) in cholesterol or M $\beta C D$-treated neurons. (D) Representative images of CTX-B and APP staining from cholesterol or M $\beta C D$-treated neurons. Scale bar $=10 \mu \mathrm{m}$. (E) Quantification of CTX-B area (left), intensity (middle), and total levels (right; area x intensity) in cholesterol or M $\beta C D$-treated neurons. (F) Quantification of APP area (left), intensity (middle), and total levels (right; area $x$ intensity) in cholesterol or M $\beta C D$-treated neurons. (G) Quantification of size and APP intensity in CTX-B/APP co-localization area. ${ }^{*} p<0.05, * * p<0.01, * * * p<0.001$ (ANOVA test followed by Dunnett's post hoc analysis, or Kruskal-Wallis test). 


\section{Supplementary Figure 3.}

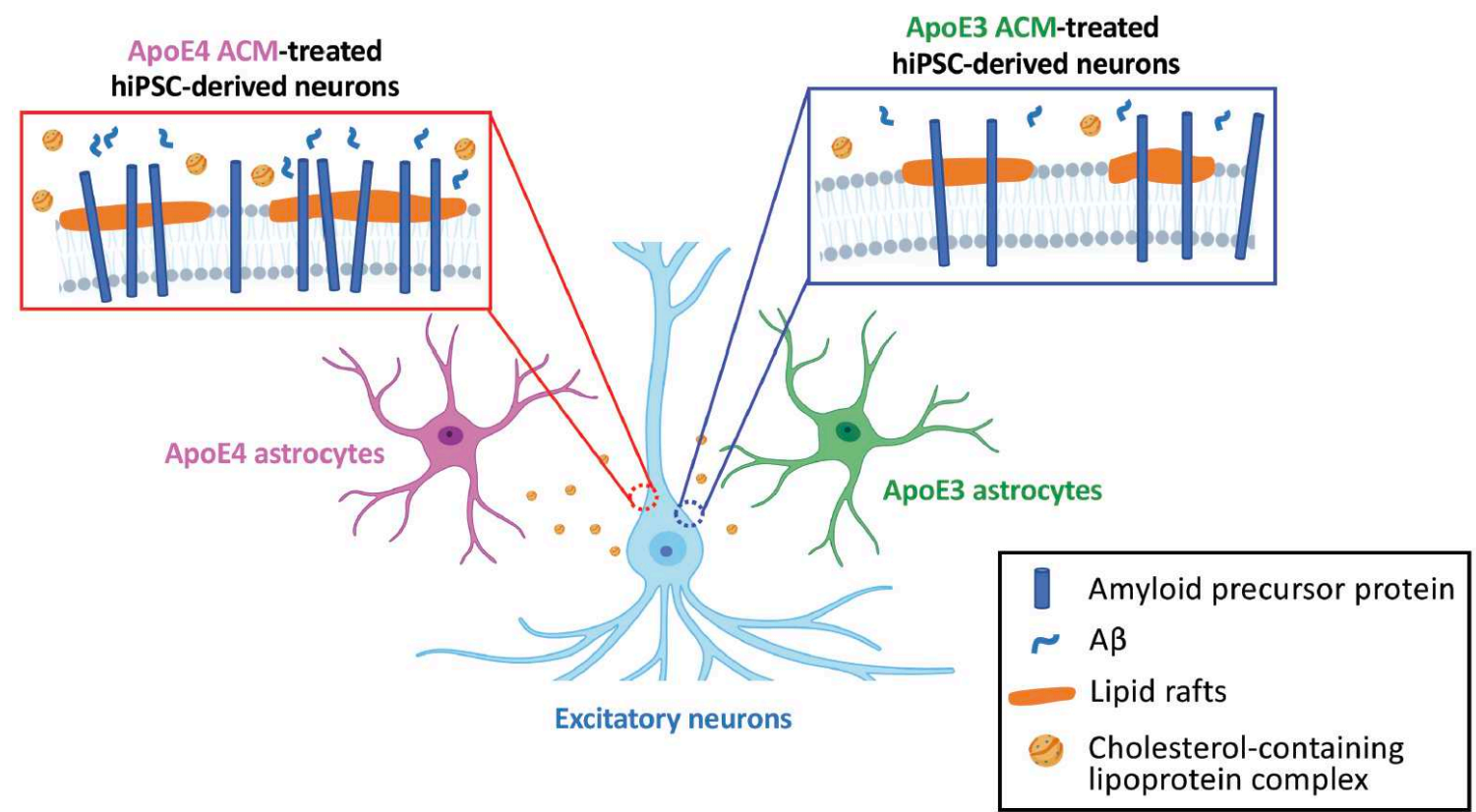




\section{Supplementary Figure 3.}

\section{Summary}

Excessive supply of cholesterol from ApoE4 astrocytes compared to ApoE3 astrocytes induces the expansion of lipid rafts, upregulation of APP, and A $\beta_{42}$ overproduction in neurons. 
A

Human iPSC-derived astrocytes

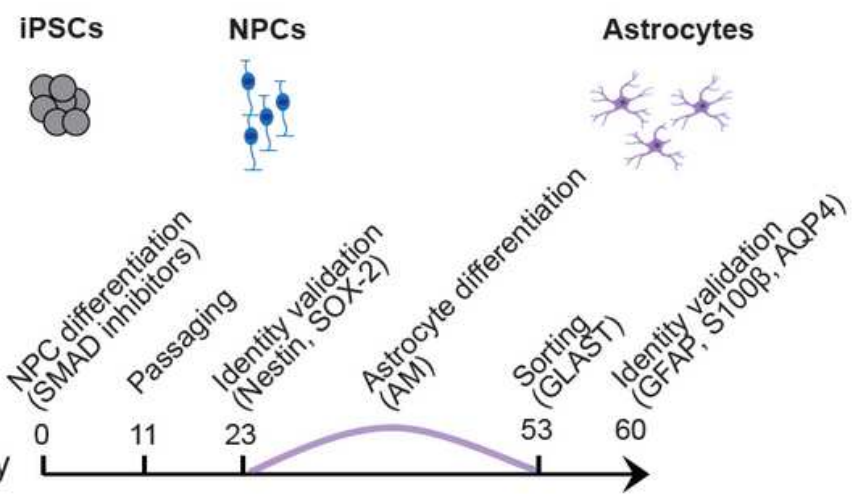

B

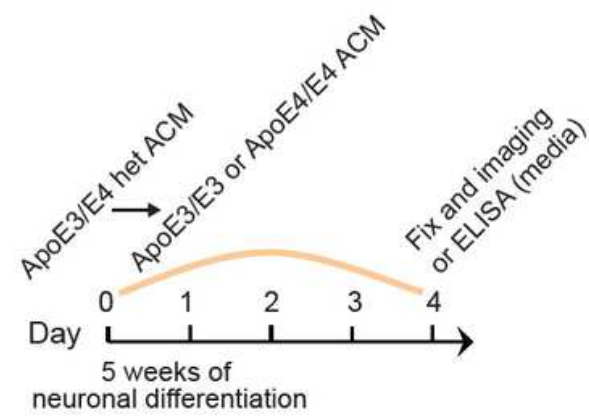

$E$

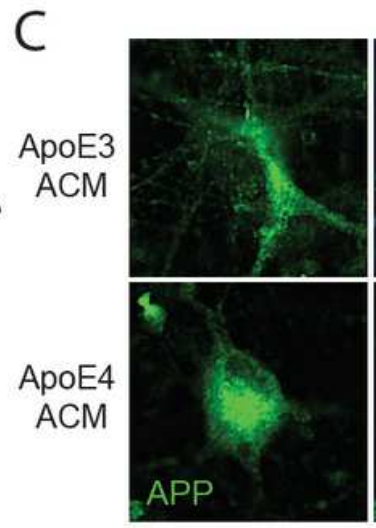

Human iPSC-derived neurons
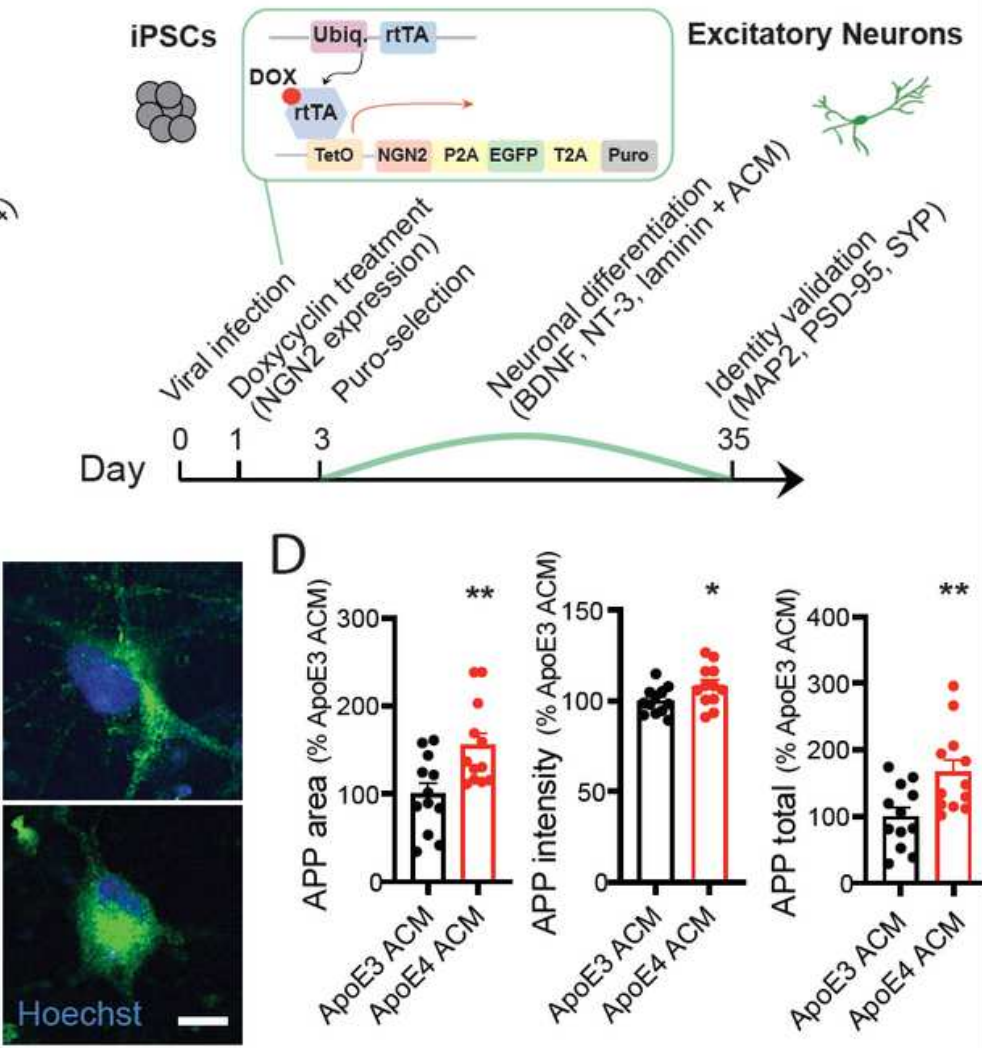

$\mathrm{F}$

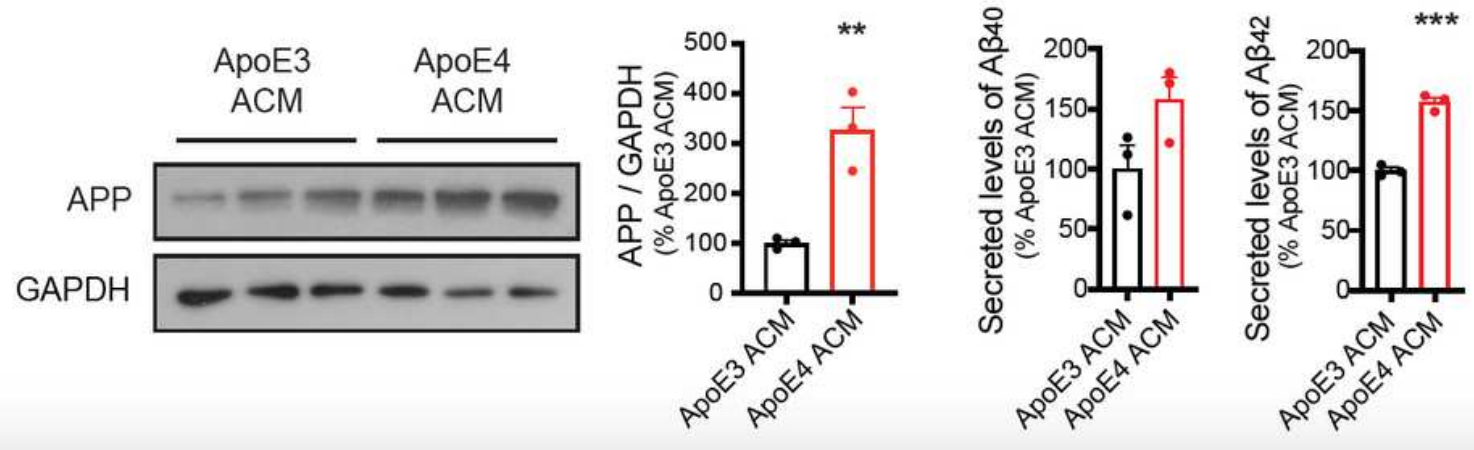

\section{Figure 1}

ApoE4 astrocyte conditioned media increase APP expression and Ab secretion in hiPSC-derived neurons.

(A) Schematics for generating astrocytes and excitatory neurons from hiPSCs. (B) Experimental procedure to examine the effect of ApoE3 or ApoE4 ACM treatment on hiPSC-derived neurons. (C) Representative images of APP staining from ACM-treated neurons. Scale bar $=10 \mu \mathrm{m}$. (D) Quantification of APP area (left), intensity (middle), and total levels (right; area x intensity) in ACM-treated neurons. (E) Western blotting for fulllength APP (APP-FL) in ApoE3 or ApoE4 ACM-treated neurons. (Right) levels of 
APP were normalized to GAPDH expression and quantified. (F) Levels of secreted $A \beta 40$ and $A \beta 42$ from ACM-treated neurons were detected by ELISA. ${ }^{*} p<0.05,{ }^{* *} p<0.01,{ }^{* *} p<0.001$ (Student's t-test).
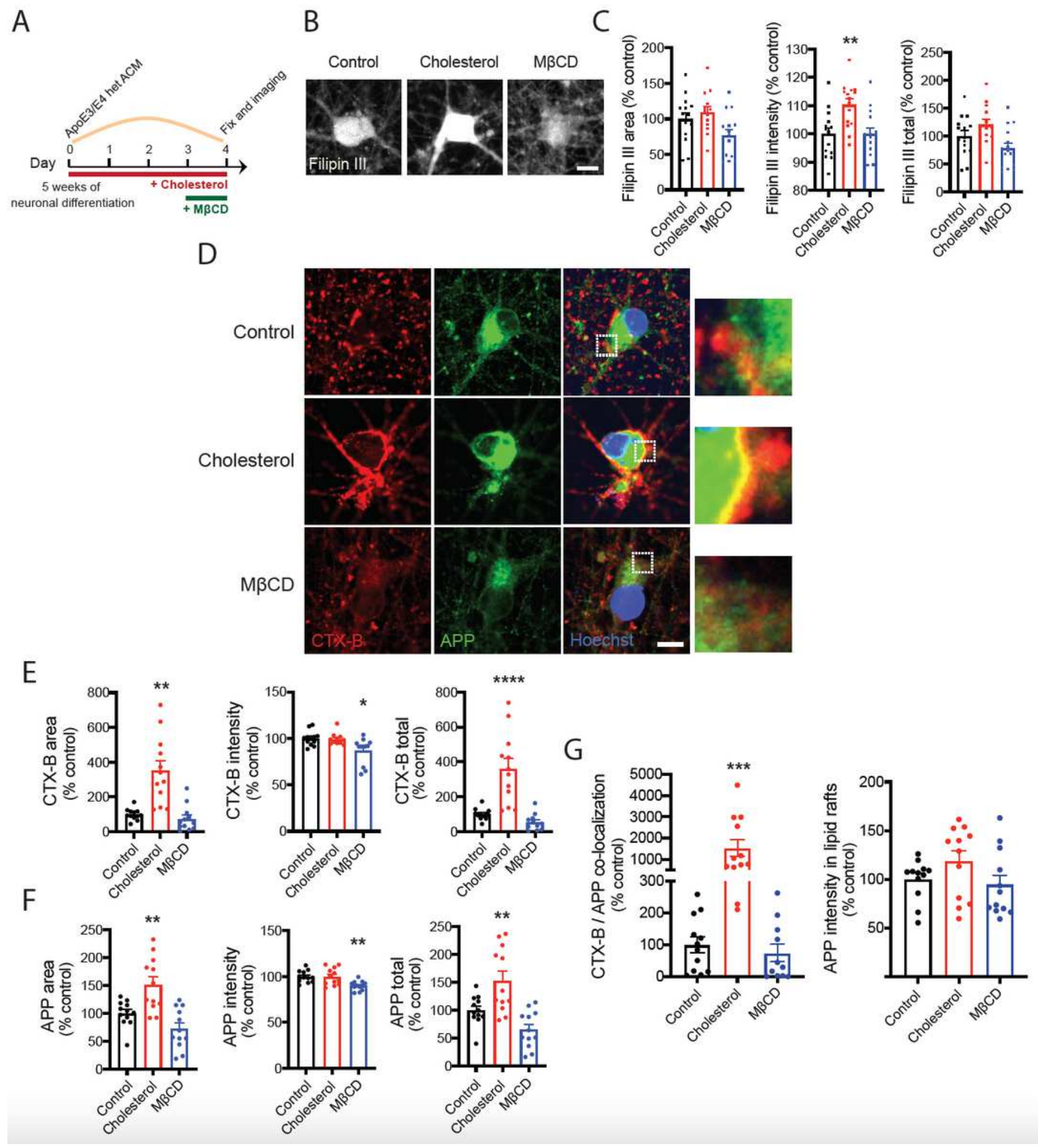

Figure 2

Cholesterol positively regulates the formation of lipid rafts and its association with APP in hiPSC-derived neurons $(A)$ Experimental procedure to examine the effect of cholesterol or M $B C D$ treatment on hiPSCderived neurons. (B) Representative images of Filipin III staining from cholesterol or M $\beta C D$-treated 
neurons. Scale bar $=10 \mu \mathrm{m}$. (C) Quantification of Filipin III area (left), intensity (middle), and total levels (right; area $\mathrm{x}$ intensity) in cholesterol or M $\beta C D$-treated neurons. (D) Representative images of CTX-B and APP staining from cholesterol or M $B C D$-treated neurons. Scale bar $=10 \mu \mathrm{m}$. (E) Quantification of CTX-B area (left), intensity (middle), and total levels (right; area $\mathrm{x}$ intensity) in cholesterol or MBCD-treated neurons. (F) Quantification of APP area (left), intensity (middle), and total levels (right; area $x$ intensity) in cholesterol or MBCD-treated neurons. (G) Quantification of size and APP intensity in CTX-B/APP colocalization area. ${ }^{*} p<0.05,{ }^{* \star} p<0.01,{ }^{* \star} p<0.001,{ }^{* \star *} p<0.0001$ (ANOVA test followed by Dunnett's post hoc analysis, or Kruskal-Wallis test).

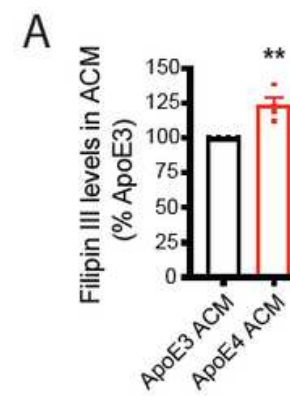

B

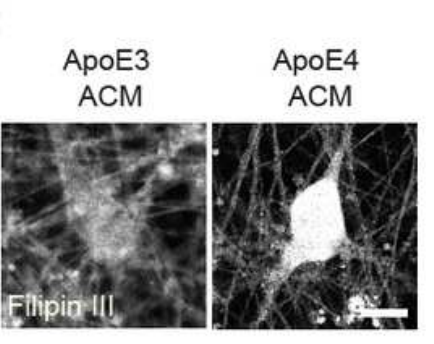

C
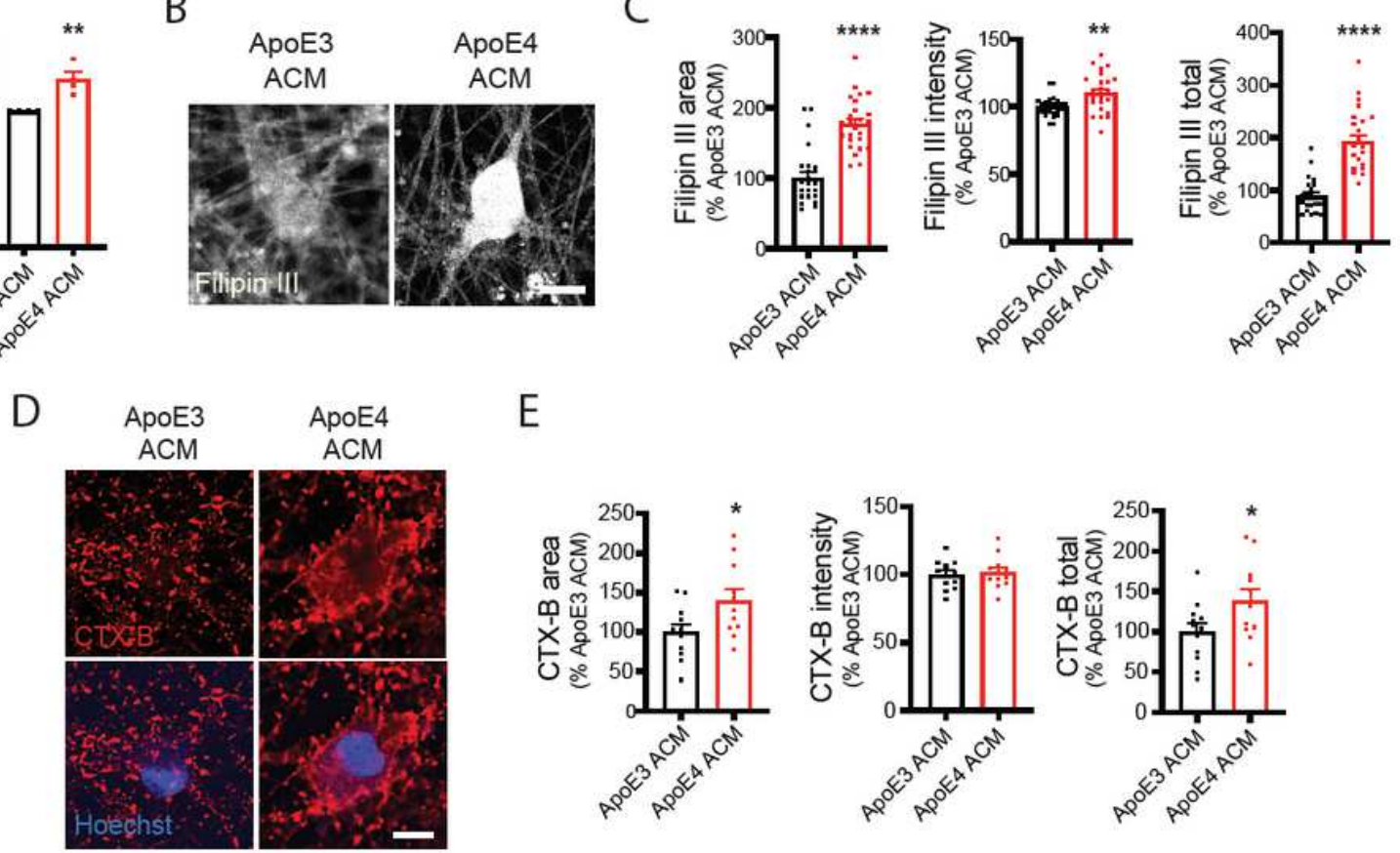

$\mathrm{E}$
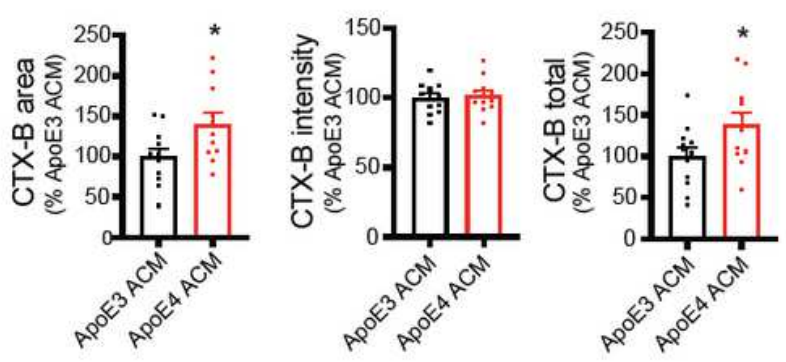

F

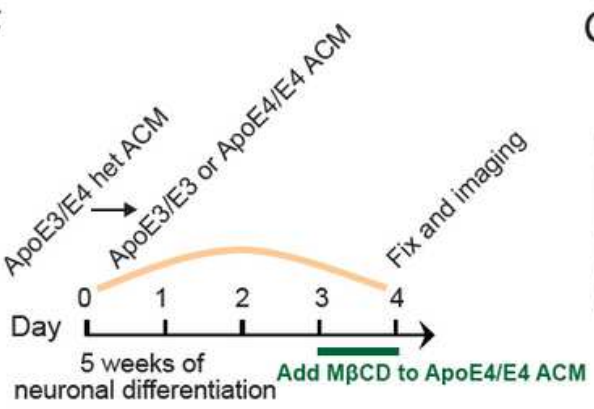

G
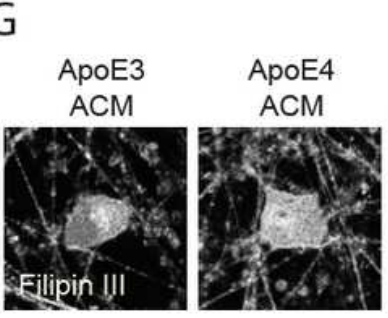

$\mathrm{H}$
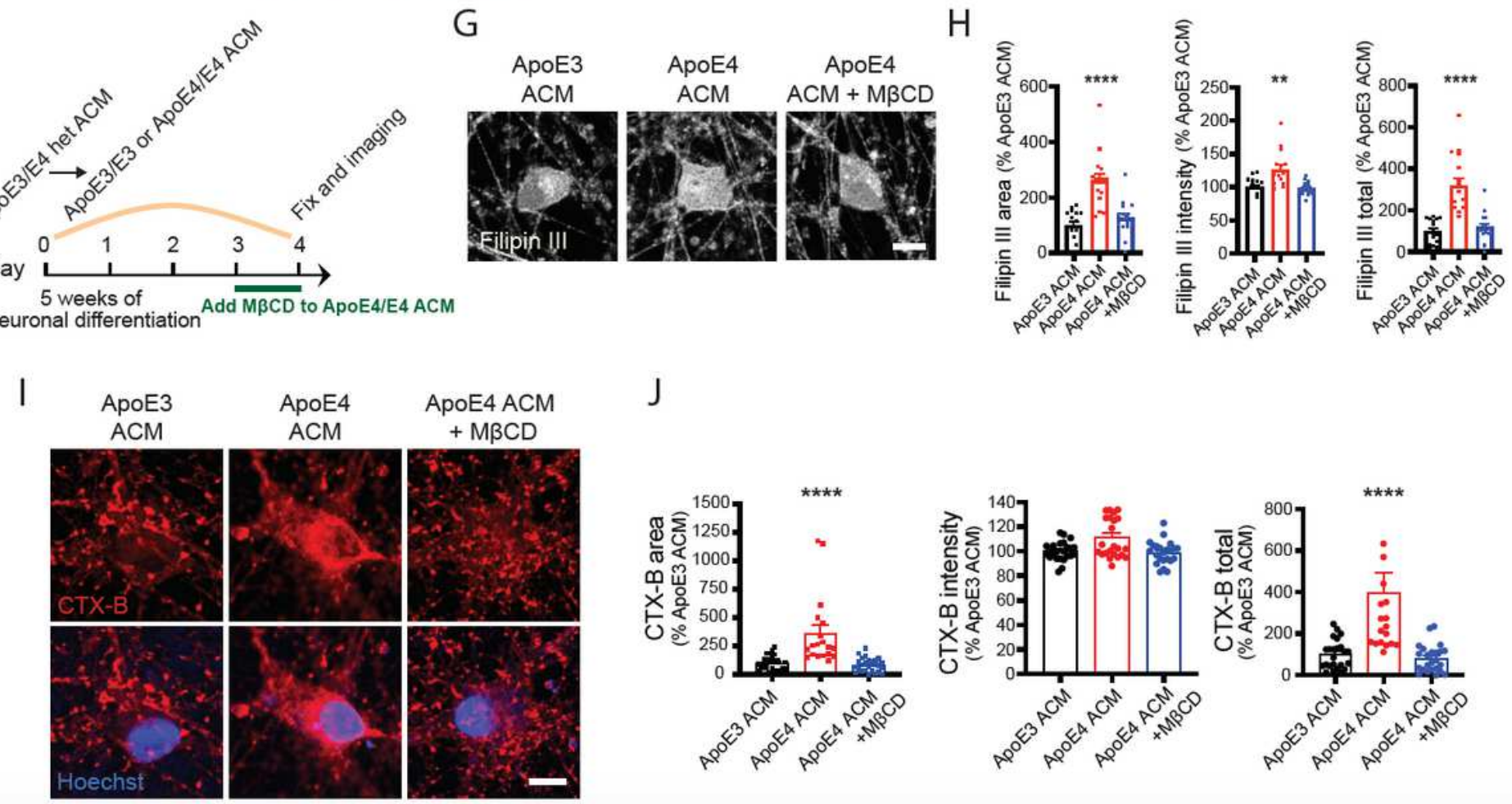

ApoE4
ACM

ApoE4 ACM

$+M B C D$
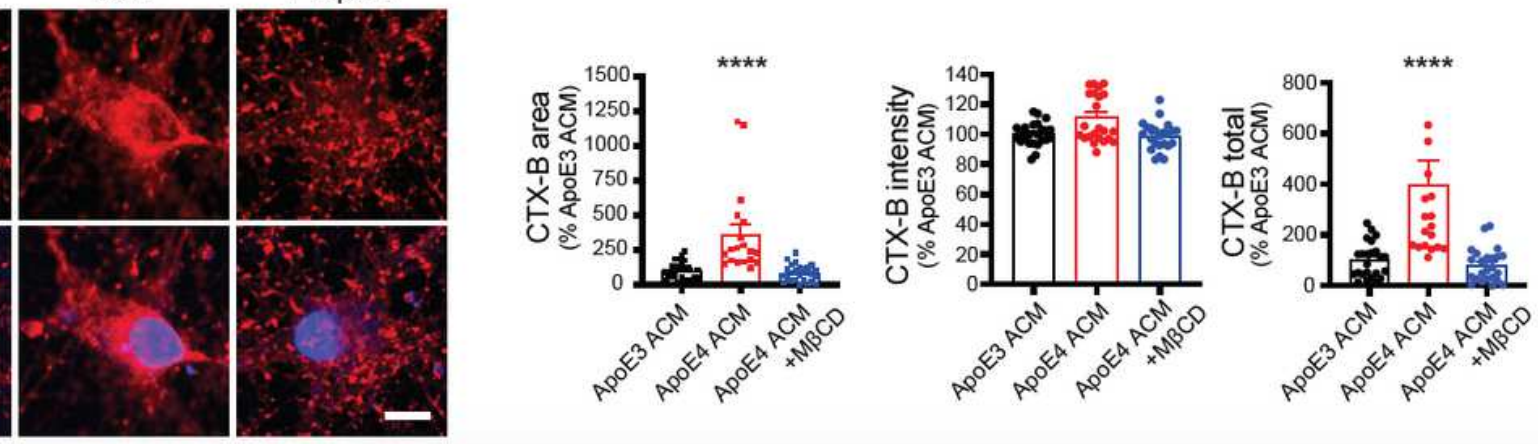


\section{Figure 3}

ApoE4 ACM increases neuronal lipid rafts (A) Measurement of secretory levels of cholesterols in ApoE3 or ApoE4 ACM via Filipin III intensity in the media. (B) Representative images of Filipin III staining from ApoE3 or ApoE4 ACM-treated neurons. Scale bar $=10 \mu \mathrm{m}$. (C) Quantification of Filipin III area (left), intensity (middle), and total levels (right; area x intensity) in ApoE3 or ApoE4 ACMtreated neurons. (D) Representative images of CTX-B staining from ApoE3 or ApoE4 ACM-treated neurons. Scale bar $=10 \mu \mathrm{m}$. (E) Quantification of CTX-B area (left), intensity (middle), and total levels (right; area x intensity) in ApoE3 or ApoE4 ACM-treated neurons. (F) Experimental procedure to examine the effect of cholesterol reduction in ApoE4 ACM on hiPSC-derived neurons. (G) Representative images of Filipin III staining from ApoE3 ACM, ApoE4 ACM, or ApoE4 ACM with MBCD-treated neurons. Scale bar $=10 \mu \mathrm{m}$. $(\mathrm{H})$ Quantification of Filipin III area (left), intensity (middle), and total levels (right; area $x$ intensity) in ApoE3 ACM, ApoE4 ACM, or ApoE4 ACM with MBCD-treated neurons. (I) Representative images of CTX-B staining from ApoE3 ACM, ApoE4 ACM or ApoE4 ACM with MBCD-treated neurons. Scale bar $=10 \mu \mathrm{m}$. (J) Quantification of CTX-B area (left), intensity (middle), and total levels (right; area $x$ intensity). ${ }^{*} p<0.05,{ }^{*} p<0.01,{ }^{\star \star \star \star \star} p<0.0001$ (Student's t-test, ANOVA test followed by Tukey's post hoc analysis, or Kruskal-Wallis test). 


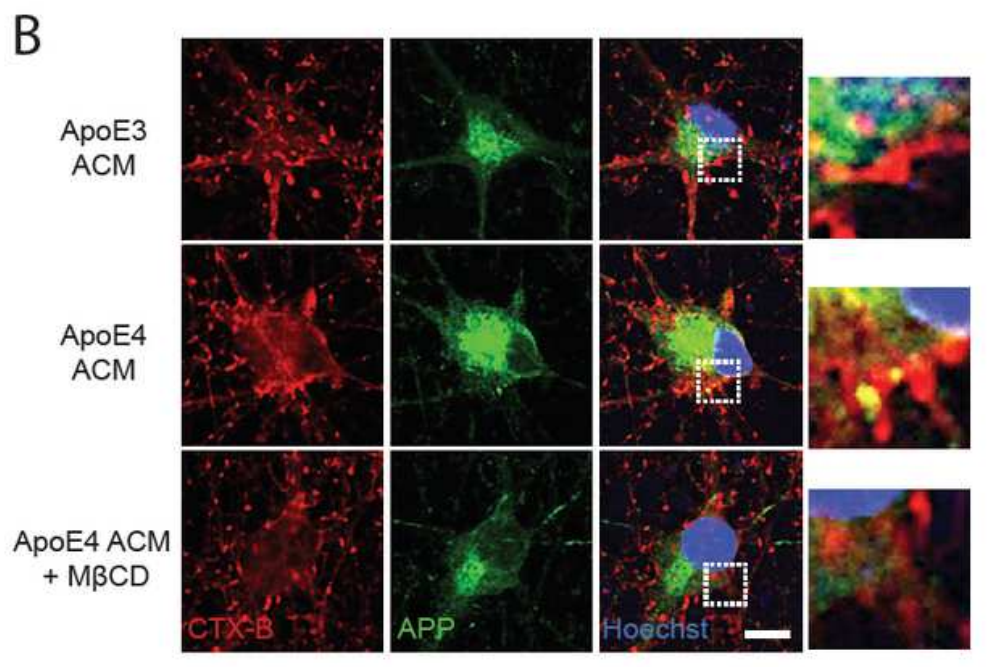

C

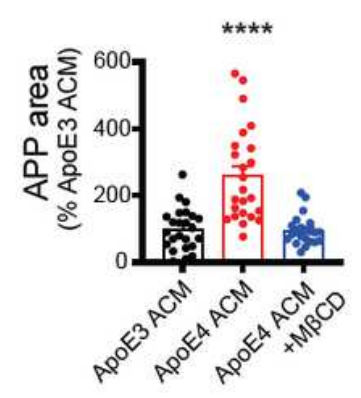

E

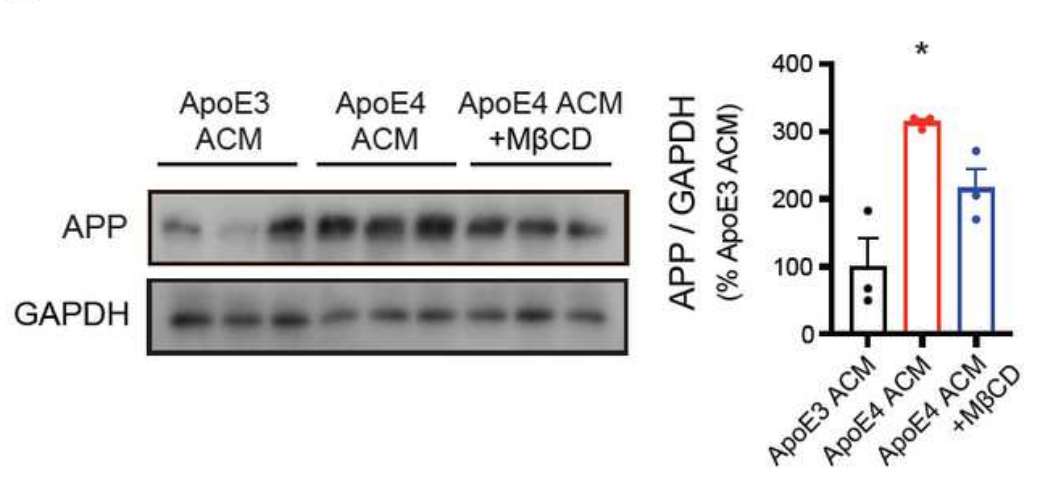

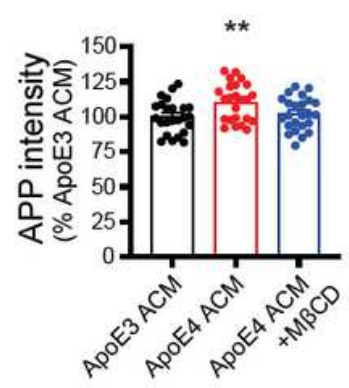

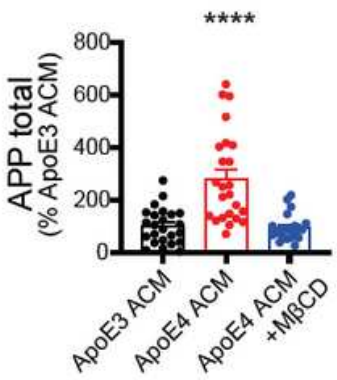

D

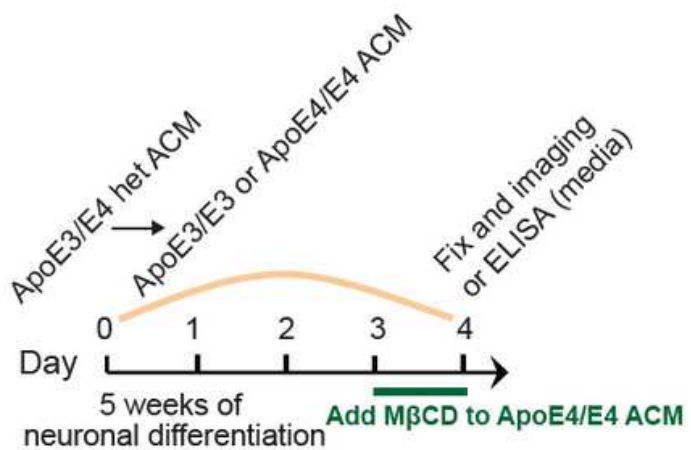

$+\mathrm{MBCD}$
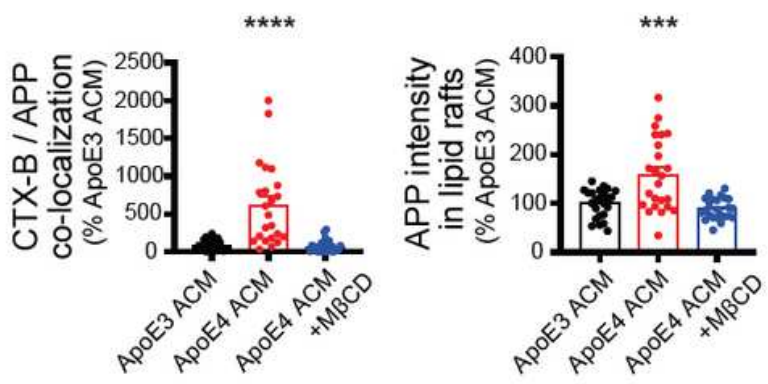

F

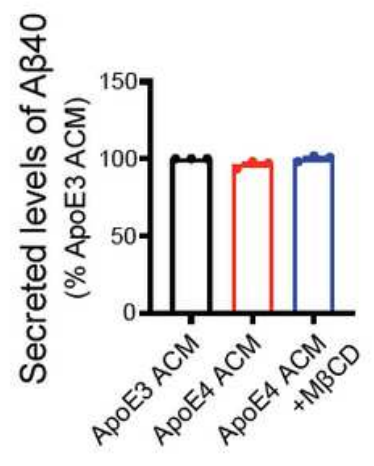

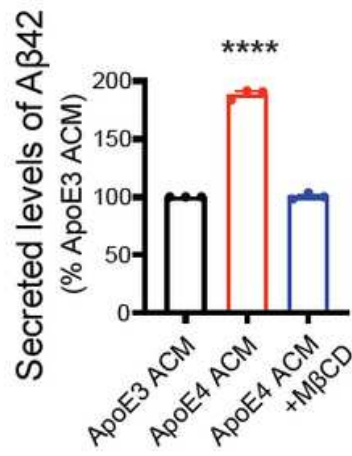

\section{Figure 4}

ApoE4 ACM increases neuronal APP expression and A $\beta$ secretion (A) Experimental procedure to examine the effect of cholesterol reduction in ApoE4 ACM on hiPSC-derived neurons. (B) Representative images of APP staining from ApoE3 ACM, ApoE4 ACM, or ApoE4 ACM with M $\beta C D$-treated neurons. Scale bar =

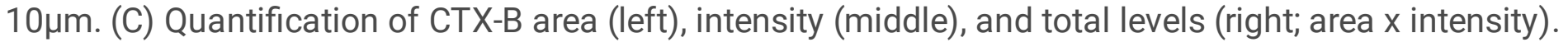
(D) Quantification of APP area (left), intensity (middle), and total levels (right; area $\mathrm{x}$ intensity). Western blotting for full-length APP (APP-FL) in ApoE3 ACM, ApoE4 ACM, or ApoE4 ACM with MßCDtreated neurons. (Right) levels of APP were normalized to GAPDH expression and quantified. (F) Levels of secreted $A \beta 40$ and $A \beta 42$ from ACM-treated neurons were detected by ELISA. * $p<0.05$, ** $p<0.01$, ${ }^{* * *} p<0.001, * \star \star \star p<0.0001$ (Student's t-test, ANOVA test followed by Dunnett's post hoc analysis, or Kruskal-Wallis test). 


\section{Supplementary Files}

This is a list of supplementary files associated with this preprint. Click to download.

- Leeetalsupplementaryinformation.pdf 